\title{
Hyperspectral Image Classification With Context-Aware Dynamic Graph Convolutional Network
}

\author{
Sheng Wan, Chen Gong, Member, IEEE, Ping Zhong, Senior Member, IEEE, Shirui Pan, Guangyu Li, \\ and Jian Yang, Member, IEEE
}

\begin{abstract}
In hyperspectral image (HSI) classification, spatial context has demonstrated its significance in achieving promising performance. However, conventional spatial context-based methods simply assume that spatially neighboring pixels should correspond to the same land-cover class, so they often fail to correctly discover the contextual relations among pixels in complex situations, and thus leading to imperfect classification results on some irregular or inhomogeneous regions such as class boundaries. To address this deficiency, we develop a new HSI classification method based on the recently proposed Graph Convolutional Network (GCN), as it can flexibly encode the relations among arbitrarily structured non-Euclidean data. Different from traditional GCN, there are two novel strategies adopted by our method to further exploit the contextual relations for accurate HSI classification. First, since the receptive field of traditional GCN is often limited to fairly small neighborhood, we proposed to capture long range contextual relations in HSI by performing successive graph convolutions on a learned regioninduced graph which is transformed from the original 2D image grids. Second, we refine the graph edge weight and the connective relationships among image regions by learning the improved adjacency matrix and the 'edge filter', so that the graph can be gradually refined to adapt to the representations generated by each graph convolutional layer. Such updated graph will in turn result in accurate region representations, and vice versa. The experiments carried out on three real-world benchmark datasets demonstrate that the proposed method yields significant improvement in the classification performance when compared with some state-of-the-art approaches.
\end{abstract}

Index Terms-Hyperspectral image classification, graph convolutional network, contextual relations, graph updating.

\section{INTRODUCTION}

$\mathbf{H}$ YPERSPECTRAL image (HSI) has recently received considerable attention in a variety of applications such as military target detection, mineral identification, and disaster prevention [1]. In contrast to traditional panchromatic and multispectral remote sensing images, HSI consists of hundreds of

S. Wan, C. Gong, G. Li, and J. Yang are with the PCA Lab, the Key Laboratory of Intelligent Perception and Systems for High-Dimensional Information of Ministry of Education, the Jiangsu Key Laboratory of Image and Video Understanding for Social Security, and the School of Computer Science and Engineering, Nanjing University of Science and Technology, Nanjing, 210094 P.R. China. (e-mail: wansheng315@ hotmail.com; chen.gong@njust.edu.cn; liguangyu0627@hotmail.com; csjyang@njust.edu.cn).

P. Zhong is with the National Key Laboratory of Science and Technology on ATR, National University of Defense Technology, Changsha 410073, China (e-mail: zhongping@ @udt.edu.cn).

S. Pan is with Faculty of Information Technology, Monash University, Clayton, VIC 3800, Australia (Email: shirui.pan@monash.edu).

Corresponding author: Chen Gong. contiguous spectral bands, which are helpful to distinguishing the targets with different materials. Thanks to the high spectral resolution, HSI has shown its advantages in identifying various land-cover types or targets [2].

Up to now, significant efforts have been made in developing diverse kinds of HSI classification methods. The earlystaged algorithms are mainly based on the simple combination of spectral signatures and conventional pattern recognition methods, such as nearest neighbor classifier and Support Vector Machines (SVM) [3], [4]. However, these methods isolatedly classify each image pixel without considering the spatial correlation among the pixels, so they will encounter the spectral variability problem [5] and generate imperfect classification results.

To address this shortcoming, the spatial context naturally becomes another type of useful information in addition to the spectra. It is now commonly acknowledged that the introduction of spatial context offers probability to improve HSI classification results and is the key to generating discriminative features for classification [6], hence there is a huge demand for the algorithms which can effectively discover and incorporate spatial context. During the past decades, researchers have reported various HSI classification methods utilizing spatial context. The first attempt was accomplished by Landgrebe and Kettig [7], where the well-known ECHO classifier is proposed to extract contextual information. After that, Markov Random Field (MRF) [8], which is an undirected graphical model [9], became a popular approach to include spatial context for HSI classification. For instance, in [10], a relative homogeneity index for each pixel is introduced in MRF-based classification to determine an appropriate weighting coefficient for the contextual contribution. Apart from this, a novel framework combining Support Vector Machine (SVM) and MRF is proposed for contextual HSI classification [11]. Meanwhile, many other models, like mathematical morphology, Gabor filtering, have also emerged as powerful tools for integrating the spatial context of HSI. For example, Benediktsson et al. [12] constructed extended morphological profiles based on the use of opening and closing morphological transforms for preprocessing HSI. Besides, in [13], a set of complex Gabor wavelets with different frequencies and orientations are designed to represent the spatial context of pixels. Additionally, multiple kernel learning [14] based on spatial context is proposed to improve the classification performance of SVM classifier on hyperspectral data. Although the use of spatial 
context improves classification results in smooth image areas [15], the aforementioned methods do not explicitly investigate the contextual relations among individual pixels (or regions), and they only implicitly assume that nearby pixels have a large probability to take the same class label regardless whether they are in object boundary regions or homogeneous regions [16]. As a result, the semantic meaning carried by image patches cannot be well preserved, and the classification errors may appear within the object area of a certain class. Additionally, the pixels around some irregular or inhomogeneous regions are also very likely to be misclassified due to the inappropriate utilization of local contextual information. Consequently, the simple assumption of smoothness and homogeneity over the whole image is unreasonable.

In order to effectively and precisely exploit the contextual relations in HSI, in this paper, we propose a novel 'ContextAware Dynamic Graph Convolutional Network' (CAD-GCN) which includes the following three key techniques: (1) The incorporation of Graph Convolutional Network (GCN) for sufficiently exploiting contextual relations among pixels; (2) The employment of graph projection and re-projection framework for exploring long range contextual relations; and (3) The utilization of dynamic graph refinement for accurately characterizing contextual relations and timely finding precise region representations.

Specifically, in our CAD-GCN, the recently proposed GCN [17] is utilized. GCN is the extension of Convolutional Neural Network $(\mathrm{CNN})$ for the non-grid data, and is able to aggregate features and propagate information across graph nodes. Consequently, the convolution operation of GCN is adaptively dominated by the neighborhood structure and can be applied to the non-Euclidean irregular data based on the graph which encodes contextual relations among graph nodes. As a result, the complex regions such as target boundaries in HSI can be flexibly preserved by GCN.

Since graph convolution is usually conducted based on neighborhood structure, its receptive field is merely limited to a fairly small region, and thus it fails to capture long range contextual relations among faraway pixels. Although constructing a dense fully-connected graph can address this deficiency, the computational cost will become unbearably heavy [18], [19]. Meanwhile, deep stacking of local operations for creating a large receptive field with long range context has also been proven inefficient [20]. Different from above approaches, by learning to project the original HSI into a region-induced graph, our proposed CAD-GCN moves beyond regular squared image grids and encodes contextual relations among regions. Then inference can be performed on the graph through passing messages between regions and along the edges connecting them. Therefore, this inference can not only update the region features, but also connect the regions which are originally far away in the 2D space by successive graph convolutions. As a result, the long range relations between faraway image regions can be effectively exploited. After that, the proposed CAD-GCN can learn an efficient graph representation with only a small number of nodes. Finally, the learned region-level features can be interpolated into the 2D feature map by reverting the pixel-to-region assignment from the previous graph projection step, so that the pixellevel features can be obtained to fully comply with the existing networks.

Nevertheless, the contextual relations revealed by a predefined fixed graph [17] for implementing GCN is still inadequate for HSI classification. Since the predefined graph based on the Euclidean distance may not be suitable for measuring their real similarities [21], we aim to learn the improved similarity measurement between image regions, in order to better characterize the contextual relationships among them. Specifically, the graph can be dynamically updated to adapt to the region representations generated by each graph convolutional layer, which will in turn make the representations more accurate. Meanwhile, since the learned graph may contain improper inter-class connections, especially around the boundaries between the regions of different classes, we introduce the 'edge filter' which can filter out the incorrect inter-class edges and refine the contextual relations represented by the graph. Intensive experimental results on three typically used HSI datasets reveal the superiority of the proposed CADGCN when compared with the exiting methods.

\section{RELATED WORKS}

In this section, we review some representative works on HSI classification and GCN, as they are related to this work.

\section{A. Hyperspectral Image Classification}

As a traditional yet critical technique, HSI classification has been an active research topic in the field of remote sensing. During the past few decades, diverse kinds of methods have been developed for HSI classification, which can be roughly divided into two categories: spectral-based methods and spectral-spatial-based methods. Many classical HSI classification approaches are only based on spectral information [3], [22] and ignore the crucial spatial information contained in HSI, which may decrease the classification performance [9]. As a result, spatial context, which has been observed to be arguably more effective than spectral signatures [6], [23], has been incorporated, to acquire better classification results. In the following, several typical methodologies for capturing the spectral-spatial information of HSI are reviewed:

1) Structural filtering. Structural Filtering-based methods have been widely studied for spatial preprocessing of HSI, where spatial features are often generated via structural filtering. Many researchers have been working on this direction, and one of the most simple but effective ways is to extract spatial information from a given region based on the moment criteria, such as the mean or standard deviation of adjacent pixels in a window [5]. The inclusion of moments or cumulants has been intensively investigated in the field of composite kernel learning and multiple kernel learning [24], [25]. Meanwhile, the adaptations of neighboring moments or cumulants have been widely adopted for HSI classification to explore the spatial homogeneity, which can preserve the image details as well [26]. In additional to structural filtering, another 
research direction is to perform local harmonic analysis, which includes spatial translation-invariant spectralspatial wavelet features, spectral-spatial Gabor features, and empirical mode decomposition-based features [27], [28]. Besides, another trend in spatial filtering is to extract features with adaptive structures, such as adaptive regionbased filtering [29].

2) Mathematical morphology. Mathematical morphology is a powerful tool for analyzing and processing geometrical structures in spatial domain [30]. In [31], the morphological profile (MP) is introduced for classifying images with very high spatial resolutions by using a sequence of geodesic opening and closing operations. Furthermore, Benediktsson et al. [12] developed the Extended MP (EMP), where an MP is computed on each component after reducing the dimensionality of data. However, the method in [12] does not fully exploit the spectral information of hyperspectral data. In order to overcome this deficiency, Fauvel et al. [32] proposed a spectral and spatial fusion approach based on EMP and the original data. Meanwhile, by extending the concept of MP and EMP, Attribute Profile (AP) [33] was proposed to extract additional spatial features for HSI classification. Since then, AP and its extensions, including extended AP [34] and extended multi-attribute profiles [35], have attracted increasing attention in HSI classification.

3) Superpixel establishment. In HSI, each superpixel corresponds to a cluster of spatially connected and spectrally similar pixels. The superpixels can also be regarded as small local regions with diverse shapes and sizes. Recently, some works focus on developing segmentationbased methods for HSI classification with superpixel technique [36], [37], in order to jointly combine the spectral-spatial correlations and discrimination to improve classification performance. For instance, in [38], superpixel technique is utilized to generate homogeneous region before constructing a graph on superpixels, which produces satisfactory classification results.

\section{B. Graph Convolutional Network}

Following the practice of conventional CNN, the concept of graph convolution can be defined by spatial methods in the vertex domain. Concretely, the convolution is defined as a weighted average function over the neighbors of each node, where the function characterizes the impact exerting to the target node from its neighboring nodes. For instance, in GraphSAGE [39], the weighting function is built by using various aggregators over the neighboring nodes. Besides, graph attention network [40] proposes to learn the weighting function via self-attention mechanism. For spatial methods, one of the open challenges is how to construct an appropriate neighborhood for the target node when defining graph convolution.

In additional to spatial methods, graph convolution can also be defined by spectral methods via convolution theorem. As the pioneering work of spectral methods, spectral CNN [41] converts signals defined in vertex domain into spectral domain by leveraging graph Fourier transform, where the convolution kernel is taken as a set of learnable coefficients associated with Fourier bases (i.e., the eigenvectors of Laplacian matrix). However, this method depends on the eigendecomposition of Laplacian matrix, which will lead to extremely high computational complexity on large-scale graphs. Subsequently, ChebyNet [42] considers the convolution kernel as a polynomial function of the diagonal matrix containing the eigenvalues of Laplacian matrix. Afterwards, GCN [17] was proposed by Kipf and Welling via using a localized first-order approximation to ChebyNet, which brings about more efficient filtering operations than spectral CNN.

Recently, GCN gains remarkable success in processing graph-structured data and has been widely adopted in many areas, such as social network mining [43], recommendation system [44], natural language processing [45], and scene understanding [46]. Due to the effectiveness of GCN in handling the non-Euclidean data, we plan to employ GCN to capture the contextual relations among pixels in HSI. To the best of our knowledge, only one prior work has employed GCN for HSI classification, i.e., [47]. However, [47] only utilizes the original fixed structure of GCN, and thus the intrinsic relations among pixels cannot be precisely explored. Moreover, the limited receptive field will degrade the convolution performance in HSI. To address these problems, we proposed a novel contextaware dynamic GCN which dynamically refines the graph along with graph convolution process and captures long range contextual relations among the image pixels via using a graph projection technique. As a result, an improved graph representation can be learned, and the performance of HSI classification can be enhanced as well.

\section{ThE PRoposed Method}

This section details the proposed CAD-GCN model, of which the pipeline is presented in Fig. 1. Given an input image (Fig. 11.a)), we first obtain its region features (Fig. 11.b)) by learning to project the original image with $2 \mathrm{D}$ pixel grids into graph data. Then dynamic graph convolution (Fig. 1.c) and Fig. 1(d)) is conducted to refine the acquired region graph, along with encoding features for each region. Finally, the classification result (Fig. 1(e)) is produced by interpolating the learned graph representation into $2 \mathrm{D}$ grids based on the regionto-pixel assignment. The critical operations in the proposed CAD-GCN will be detailed by presenting the GCN backbone (Section III-A), explaining the graph projection with pixelto-region assignment (Section III-B), describing the dynamic graph refinement (Section III-C), and elaborating the graph re-projection with region-to-pixel assignment (Section III-D).

\section{A. Graph Convolutional Network}

Inspired by $\mathrm{CNN}, \mathrm{GCN}$ [17] is a multi-layer neural network which directly operates on a graph and aims to extract highlevel features through aggregating feature information from the neighborhoods of graph nodes. In GCN, an undirected graph is formally defined as $\mathcal{G}=(\mathcal{V}, \mathcal{E})$ with $\mathcal{V}$ and $\mathcal{E}$ denoting the sets of nodes and edges, respectively. The notation $\mathbf{A}$ denotes the adjacency matrix of $\mathcal{G}$ which indicates the existence of an 


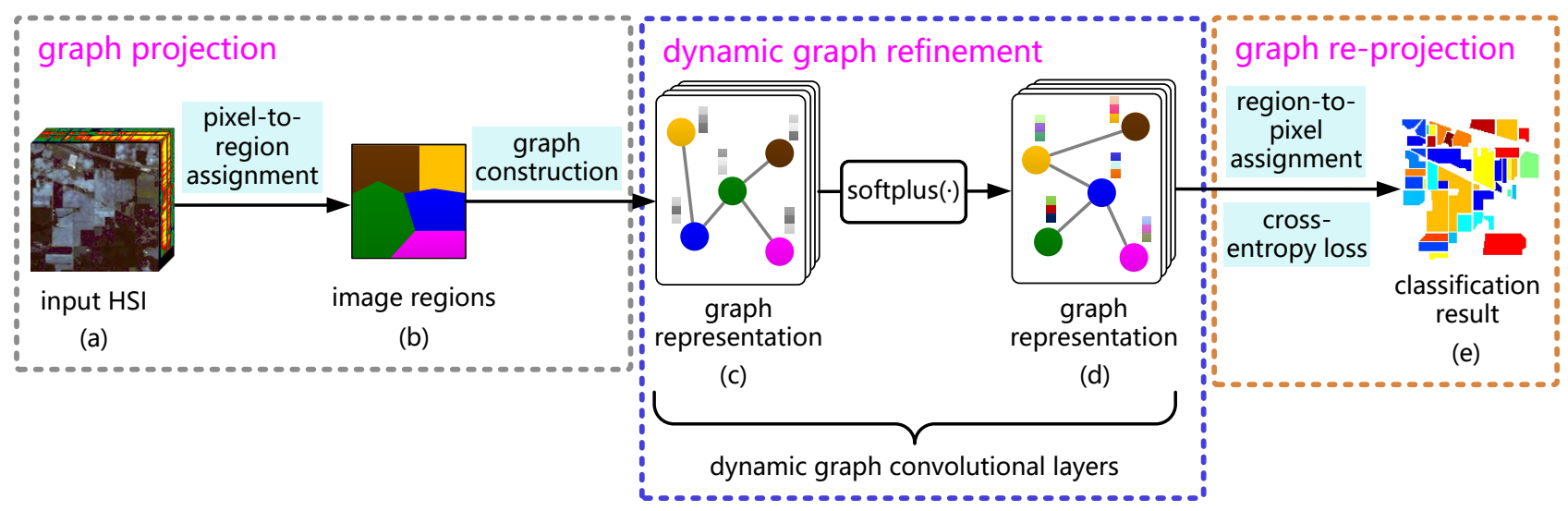

Fig. 1. The framework of our proposed CAD-GCN. (a) is the original HSI. (b) shows the five regions from the original HSI obtained via pixel-to-region assignment. (c) and (d) denote two dynamic graph convolutional layers, where the circles with different colors correspond to different image regions (i.e., graph nodes) and the gray lines represent graph edges. From (c) to (d), both the edge weight and the connective relationships among regions can be dynamically refined during the convolution operation, and thus the improved graph structure and node representations can be obtained. In our model, softplus [48] is utilized as the activation function. In (e), the learned graph representation can be interpolated back into 2D image grids based on the region-to-pixel assignment, and then the cross-entropy loss is used to penalize the label differences between the network output and the originally labeled pixels.

edge between each pair of nodes, and its $(i, j)^{\text {th }}$ element can be calculated as

$$
\mathbf{A}_{i j}= \begin{cases}e^{-\gamma\left\|\mathbf{x}_{i}-\mathbf{x}_{j}\right\|^{2}} & \text { if } \mathbf{x}_{i} \in N\left(\mathbf{x}_{j}\right) \text { or } \mathbf{x}_{j} \in N\left(\mathbf{x}_{i}\right) \\ 0 & \text { otherwise }\end{cases}
$$

where the parameter $\gamma$ is empirically set to 0.2 in our experiments, $\mathbf{x}_{i}$ and $\mathbf{x}_{j}$ represent two graph nodes (i.e., image regions in this paper), and $N\left(\mathbf{x}_{j}\right)$ is the set of neighbors of $\mathbf{x}_{j}$.

First, in order to conduct node embedding for $\mathcal{G}$, spectral filtering on the graph is defined, which can be expressed as the multiplication of a signal $\mathbf{x}$ with a filter $g_{\boldsymbol{\theta}}=\operatorname{diag}(\boldsymbol{\theta})$ in the Fourier domain, i.e.,

$$
g_{\boldsymbol{\theta}} \star \mathbf{x}=\mathbf{U} g_{\boldsymbol{\theta}} \mathbf{U}^{\top} \mathbf{x},
$$

where $\mathbf{U}$ is the matrix of eigenvectors of normalized graph Laplacian $\mathbf{L}=\mathbf{I}-\mathbf{D}^{-\frac{1}{2}} \mathbf{A} \mathbf{D}^{-\frac{1}{2}}=\mathbf{U} \boldsymbol{\Lambda} \mathbf{U}^{\top}$. Here $\boldsymbol{\Lambda}$ denotes a diagonal matrix composed of the eigenvalues of $\mathbf{L}, \mathbf{D}$ is the degree matrix with the diagonal element $\mathbf{D}_{i i}=\sum_{j} \mathbf{A}_{i j}$, and $\mathbf{I}$ represents the identity matrix with proper size throughout this paper. Then $g_{\boldsymbol{\theta}}$ can be understood as a function of eigenvalues of $\mathbf{L}$, i.e., $g_{\boldsymbol{\theta}}(\boldsymbol{\Lambda})$. To reduce the computational cost of eigendecomposition in Eq. (2), Hammond et al. [49] approximated $g_{\boldsymbol{\theta}}(\boldsymbol{\Lambda})$ using a truncated expansion in terms of Chebyshev polynomials $T_{k}(\mathbf{x})$ up to $K^{\text {th }}$-order, namely

$$
g_{\boldsymbol{\theta}^{\prime}}(\boldsymbol{\Lambda}) \approx \sum_{k=0}^{K} \boldsymbol{\theta}_{k}^{\prime} T_{k}(\widetilde{\boldsymbol{\Lambda}})
$$

where $\boldsymbol{\theta}^{\prime}$ denotes a vector of Chebyshev coefficients, and $\widetilde{\boldsymbol{\Lambda}}=\frac{2}{\lambda_{\max }} \boldsymbol{\Lambda}-\mathbf{I}$ with $\lambda_{\max }$ being the largest eigenvalue of $\mathbf{L}$. According to [49], the Chebyshev polynomials can be defined as $T_{k}(\mathbf{x})=2 \mathbf{x} T_{k-1}(\mathbf{x})-T_{k-2}(\mathbf{x})$, where $T_{0}(\mathbf{x})=1$ and $T_{1}(\mathbf{x})=\mathbf{x}$. Hence, we have the convolution of a signal $\mathbf{x}$ as

$$
g_{\boldsymbol{\theta}^{\prime}} \star \mathbf{x} \approx \sum_{k=0}^{K} \boldsymbol{\theta}_{k}^{\prime} T_{k}(\widetilde{\mathbf{L}}) \mathbf{x},
$$

where $\widetilde{\mathbf{L}}=\frac{2}{\lambda_{\max }} \mathbf{L}-\mathbf{I}$ is the scaled Laplacian matrix. Eq. (4) can be easily verified according to the fact that $\left(\mathbf{U} \boldsymbol{\Lambda} \mathbf{U}^{\top}\right)^{k}=$ $\mathbf{U} \Lambda^{k} \mathbf{U}^{\top}$. As is can be observed, this expression is a $K^{\text {th }}$ order polynomial regarding the Laplacian (i.e., $K$-localized). In other words, the filtering only depends on the nodes that are at most $K$ steps away from the central node. In our CADGCN model, the first-order neighborhood is considered, i.e., $K=1$, and thus Eq. (4) turns to a linear function on the graph Laplacian spectrum with respect to $\mathbf{L}$.

Afterwards, a neural network based on graph convolutions can be built by stacking multiple convolutional layers in the form of Eq. (4), where each layer is followed by an elementwise non-linear operation (i.e., softplus(.) [48]). By this way, we can derive diverse classes of convolutional filter functions through stacking multiple layers of the same configuration. With the linear formulation, Kipf and Welling [17] further approximated $\lambda_{\max } \approx 2$, considering that the network parameters can adapt to this change in scale during the training process. Therefore, Eq. (4) is simplified to

$$
g_{\boldsymbol{\theta}^{\prime}} \star \mathbf{x} \approx \boldsymbol{\theta}_{0}^{\prime} \mathbf{x}+\boldsymbol{\theta}_{1}^{\prime}(\mathbf{L}-\mathbf{I}) \mathbf{x}=\boldsymbol{\theta}_{0}^{\prime} \mathbf{x}-\boldsymbol{\theta}_{1}^{\prime} \mathbf{D}^{-\frac{1}{2}} \mathbf{A D}^{-\frac{1}{2}} \mathbf{x},
$$

where $\boldsymbol{\theta}_{0}^{\prime}$ and $\boldsymbol{\theta}_{1}^{\prime}$ are two free parameters. Since reducing the number of parameters helps to avoid overfitting, Eq. (5) is further converted to

$$
g_{\boldsymbol{\theta}} \star \mathbf{x} \approx \boldsymbol{\theta}\left(\mathbf{I}+\mathbf{D}^{-\frac{1}{2}} \mathbf{A} \mathbf{D}^{-\frac{1}{2}}\right) \mathbf{x}
$$

by letting $\boldsymbol{\theta}=\boldsymbol{\theta}_{0}^{\prime}=-\boldsymbol{\theta}_{1}^{\prime}$. As $\mathbf{I}+\mathbf{D}^{-\frac{1}{2}} \mathbf{A D}^{-\frac{1}{2}}$ has the eigenvalues in the range $[0,2]$, repeatedly applying this operator will result in numerical instabilities and exploding/vanishing gradients in a deep network. To solve this deficiency, Kipf and Welling [17] performed the re-normalization trick $\mathbf{I}+\mathbf{D}^{-\frac{1}{2}} \mathbf{A D}^{-\frac{1}{2}} \rightarrow \widetilde{\mathbf{D}}-\frac{1}{2} \widetilde{\mathbf{A}} \widetilde{\mathbf{D}}^{-\frac{1}{2}}$ with $\widetilde{\mathbf{A}}=\mathbf{A}+\mathbf{I}$ and $\widetilde{\mathbf{D}}_{i i}=\sum_{j} \widetilde{\mathbf{A}}_{i j}$. As a result, the convolution operation of GCN model can then be expressed as

$$
\mathbf{H}^{(l)}=\sigma\left(\widetilde{\mathbf{A}} \mathbf{H}^{(l-1)} \mathbf{W}^{(l)}\right),
$$

where $\mathbf{H}^{(l)}$ denotes the output of the $l^{\text {th }}$ layer, $\sigma(\cdot)$ represents an activation function, such as the softplus function [48] used 
in our proposed CAD-GCN, and $\mathbf{W}^{(l)}$ is the trainable weight matrix involved in the $l^{\text {th }}$ layer.

\section{B. Pixel-to-Region Assignment}

Although GCN is able to capture contextual relations among image pixels, the receptive field of pixel-level graph convolution is often limited [19]. In order to effectively characterize long range relations among pixels, we intend to move beyond regular 2D image grids and encode contextual relations among regions, since the dependencies among image regions are of much longer than those captured by pixel-level convolutions [19]. The main idea is learning pixel-to-region assignment which groups pixels with similar features into coherent regions, in order to capture contextual relations among the regions originally far away in the original $2 \mathrm{D}$ space.

Different from the conventional region-based methods [16], [38] which start by coarsely grouping pixels into certain regions, we aim at learning to transform the original HSI into a region graph, and this process is called graph projection. Specifically, a soft assignment matrix which is parameterized by $\mathbf{V} \in \mathbb{R}^{d \times c}$ will be learned by the network to assign each pixel $\mathbf{z}_{i} \in \mathbb{R}^{d}$ to its neighboring regions, where $d$ denotes the spectral dimensionality of each pixel, $c$ is the number of image regions, and each column $\mathbf{v}_{i} \in \mathbb{R}^{d}$ of $\mathbf{V}$ corresponds to the anchor point of a region. Then the soft assignment matrix $\mathbf{P} \in \mathbb{R}^{n \times c}$ can be computed as

$$
\mathbf{P}_{i j}= \begin{cases}e^{-\gamma\left\|\mathbf{z}_{i}-\mathbf{v}_{j}\right\|^{2}} & \text { if } \mathbf{v}_{j} \in \tilde{N}\left(\mathbf{z}_{i}\right) \\ 0 & \text { otherwise }\end{cases}
$$

where $n$ is the number of image pixels, $\widetilde{N}\left(\mathbf{z}_{i}\right)$ denotes the set of neighboring regions connected to the pixel $\mathbf{z}_{i}$. To be more specific, $\widetilde{N}\left(\mathbf{z}_{i}\right)$ includes not only the central region where $\mathbf{z}_{i}$ resides, but also the regions adjacent that are to the central one. In Eq. (8), the element $\mathbf{P}_{i j}$ defines the soft assignment of a pixel $\mathbf{z}_{i}$ to $\mathbf{v}_{j}$. With the learned pixel-to-region assignment, the region feature $\mathbf{x}_{j}$ can be encoded by

$$
\mathbf{x}_{j}=\frac{\sum_{i} \mathbf{P}_{i j} \mathbf{z}_{i}}{\sum_{i} \mathbf{P}_{i j}} .
$$

By learning the features for each region, the negative impact of inaccurate pre-computed region features can be reduced.

However, there still exists an optimization challenge, since most or even all of the image pixels may be assigned to a single region in some extreme circumstances. This is probably because that the anchor point matrix for image regions $\mathbf{V}$ is initialized improperly, which will subsequently result in an ill-posed assignment matrix $\mathbf{P}$. Moreover, the imbalanced assignment will lead to unfavorable graph structure, and thus the contextual relations cannot be sufficiently exploited. To cope with this problem, instead of initializing $\mathbf{V}$ randomly, we take the spatial information into consideration and initialize $\mathbf{V}$ by utilizing a segmentation technique. Specifically, the Simple Linear Iterative Clustering (SLIC) algorithm [50], which has been widely used for image segmentation, is employed to obtain the initial regions. Here the average spectral signatures of the pixels involved in the corresponding region will be utilized to initialize each $\mathbf{v}_{i}$, and the matrix

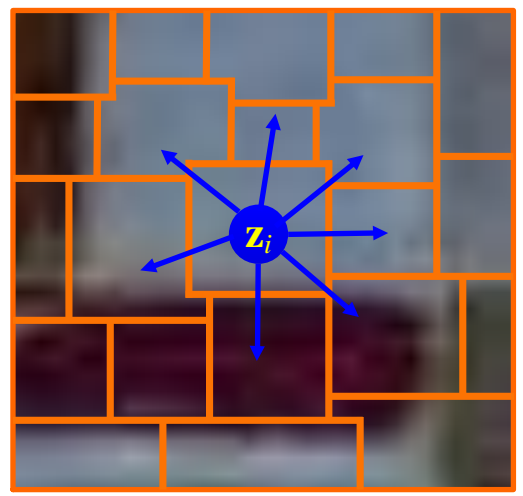

Fig. 2. Illustration of the soft pixel-to-region assignment used in our CADGCN model. Each of the initialized image regions is surrounded by yellow lines, and the blue arrows denote the assignment regarding the pixel $\mathbf{z}_{i}$ to its neighboring regions.

V can be further updated via using gradient descent. This segmentation-based initialization technique can yield more stable training performance and produce more meaningful graph representation than random initialization [19]. Fig. 2 exhibits the pixel-to-region assignment regarding a pixel $\mathbf{z}_{i}$. With the learned region features, the corresponding region graph can be naturally acquired by using Eq. (1). After that, the region features $\mathbf{X}$ will be re-computed by performing graph convolution [17] which aggregates information along the edges. Moreover, through successive graph convolutions, long range dependencies among the regions that are far away in the original 2D space can be captured.

\section{Dynamic Graph Refinement}

The performance of graph convolution largely depends on the quality of the predefined graph which encodes the similarities and connective relationships among graph nodes. However, the Euclidean distance, which is widely used for characterizing node similarities (e.g., in Eq. (1)), may not be a good metric for graph structured data [21]. In order to address this weakness, we aim to learn an improved distance metric. Specifically, we construct a symmetric positive semi-definite matrix $\mathbf{M}=\mathbf{W}_{d} \mathbf{W}_{d}^{\top}$ with $\mathbf{W}_{d}$ being a trainable weight matrix. Then the Generalized Mahalanobis distance can be formulated as follows:

$$
\mathcal{D}\left(\mathbf{x}_{i}, \mathbf{x}_{j}\right)=\sqrt{\left(\mathbf{x}_{i}-\mathbf{x}_{j}\right)^{\top} \mathbf{M}\left(\mathbf{x}_{i}-\mathbf{x}_{j}\right)} .
$$

Afterwards, the adjacency matrix $\mathbf{A}$ in Eq. (1) can be rewritten as

$$
\mathbf{A}_{i j}=\left\{\begin{array}{ll}
e^{-\gamma\left(\mathcal{D}\left(\mathbf{x}_{i}, \mathbf{x}_{j}\right)\right)^{2}} & \text { if } \mathbf{x}_{i} \in N\left(\mathbf{x}_{j}\right) \text { or } \mathbf{x}_{j} \in N\left(\mathbf{x}_{i}\right) \\
0 & \text { otherwise }
\end{array} .\right.
$$

Since the graph representation is updated along with the graph convolutional layers, learning a single matrix $\mathbf{M}$ is insufficient to accurately measure node similarities for all the layers. Therefore, we adaptively learn the symmetric positive semidefinite parameter matrix $\mathbf{M}^{(l)}$ for the adjacency matrix $\mathbf{A}^{(l)}$ which is utilized in the $l^{\text {th }}$ layer, in order to acquire the 
improved node similarities. Then Eq. (11) can be rewritten as

$$
\mathbf{A}_{i j}^{(l+1)}=\left\{\begin{array}{ll}
e^{-\gamma\left(\mathcal{D}^{(l)}\left(\mathbf{h}_{i}^{(l)}, \mathbf{h}_{j}^{(l)}\right)\right)^{2}} & \text { if } \mathbf{x}_{i} \in N\left(\mathbf{x}_{j}\right) \text { or } \\
0 & \mathbf{x}_{j} \in N\left(\mathbf{x}_{i}\right) \\
0 & \text { otherwise }
\end{array},\right.
$$

where $\mathbf{h}_{i}^{(l)}$ is the representation of $\mathbf{x}_{i}$ generated by the $l^{\text {th }}$ layer with $\mathbf{h}_{i}^{(0)}=\mathbf{x}_{i}$, and $\mathcal{D}^{(l)}\left(\mathbf{h}_{i}^{(l)}, \mathbf{h}_{j}^{(l)}\right)$ can be formulated as $\sqrt{\left(\mathbf{h}_{i}^{(l)}-\mathbf{h}_{j}^{(l)}\right)^{\top} \mathbf{M}^{(l)}\left(\mathbf{h}_{i}^{(l)}-\mathbf{h}_{j}^{(l)}\right)}$.

During graph construction, connections among the regions from different classes may be incorporated, which will lead to the aggregation of inter-class feature information and further degrade the discriminability of graph convolution results. To overcome this deficiency, we propose to use the edge filter, which aims to refine the contextual relations by reducing undesirable inter-class edges of the graph. Since the intraclass examples are generally more similar than the inter-class ones, it is believed that the element $\mathbf{A}_{i j}^{(l)}$ with relatively small value is more likely to represent inter-class relations than the $\mathbf{A}_{i j}^{(l)}$ with large value. Therefore, we employ a threshold $\beta^{(l)}$ for each graph convolutional layer to filter out the interclass relations and reduce the adverse effect of inter-class feature aggregation. The selection of $\beta^{(l)}$ will be discussed in Section IV Specifically, in the $l^{\text {th }}$ layer, the edge filter $\mathcal{F}(\cdot)$ used can be simply expressed as

$$
\mathcal{F}\left(\mathbf{A}_{i j}^{(l)}\right)=\left\{\begin{array}{ll}
\mathbf{A}_{i j}^{(l)} & \text { if } \mathcal{F}\left(\mathbf{A}_{i j}^{(l)}\right)>\beta^{(l)} \\
0 & \text { otherwise }
\end{array} .\right.
$$

In practice, constraining the number of parameters can be beneficial to address the problem of overfitting [17], and thus we set $\beta^{(l)}=\beta$ for all the layers. With the edge filter, the graph convolutional layer can then be reformulated as

$$
\mathbf{H}^{(l)}=\sigma\left(\mathcal{F}\left(\mathbf{A}^{(l)}\right) \mathbf{H}^{(l-1)} \mathbf{W}^{(l)}\right),
$$

with $\mathbf{H}^{(0)}=\mathbf{X}$.

\section{Region-to-Pixel Assignment}

After conducting the dynamic graph convolution on the region level, we need to re-project the new region features (i.e., the learned graph representation) $\mathbf{H}^{(L)}$ back into $2 \mathrm{D}$ image with grids of pixels, and this process is called graph re-projection, Specifically, the region-to-pixel assignment is accomplished by linearly interpolating pixel features based on the soft assignment matrix $\mathbf{P}$, namely $\mathbf{P} \mathbf{H}^{(L)}$, where $L$ denotes the number of graph convolutional layers. Note that all the re-projected pixels will have diverse feature representations, even if some of them are assigned to the same region. Therefore, the contextual details of the HSI can be well preserved.

With the region-to-pixel assignment, the output of our proposed CAD-GCN can be obtained as

$$
\mathbf{O}=\mathbf{P H}^{(L)} .
$$

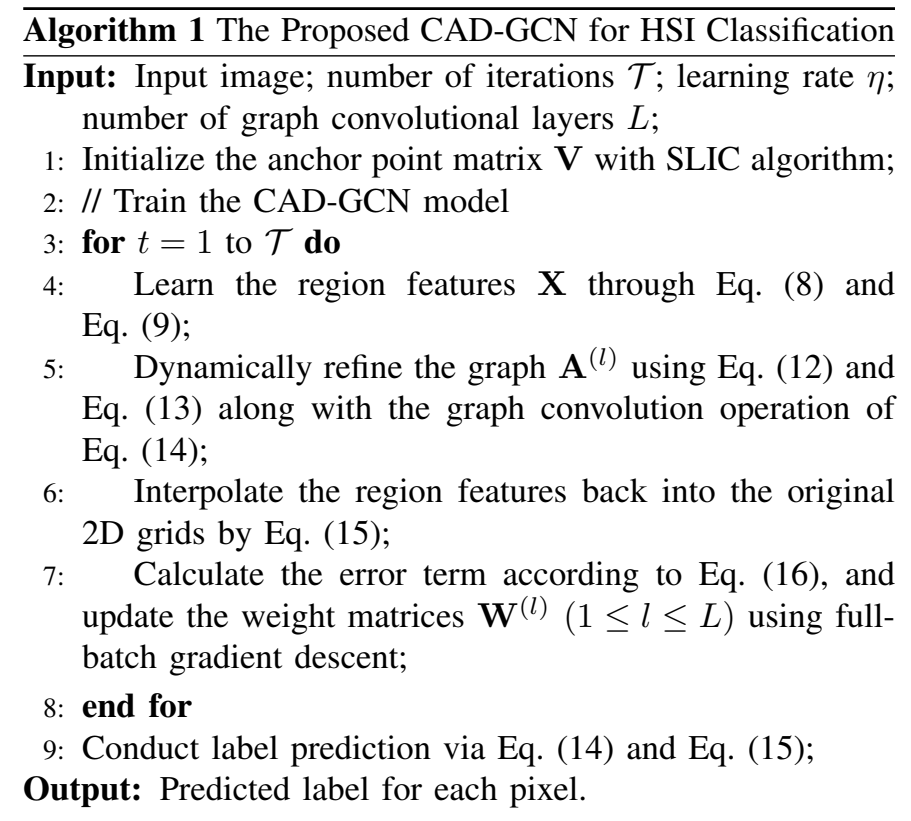

In our CAD-GCN model, the cross-entropy error is employed to penalize the differences between the network output and the labels of labeled pixels, namely

$$
\mathcal{L}=-\sum_{g \in \mathbf{y}_{G}} \sum_{f=1}^{C} \mathbf{Y}_{g f} \ln \mathbf{O}_{g f},
$$

where $C$ is the number of classes, $\mathbf{y}_{G}$ denotes the set of indices corresponding to the labeled pixels, and $\mathbf{Y}$ represents the label matrix. Here, we let $\mathbf{Y}_{i j}$ be 1 if the pixel $\mathbf{z}_{i}$ belongs to the $j^{\text {th }}$ class, and 0 otherwise. It is noticeable that our model can be trained via an end-to-end way. Similar to [17], full-batch gradient descent is utilized to update the network parameters for CAD-GCN. Algorithm 1 shows the summarization of our proposed CAD-GCN classification method.

\section{EXPERIMENTAL RESULTS}

To test the effectiveness of the proposed CAD-GCN model, in this section, we conduct exhaustive experiments on three real-world benchmark datasets, namely Indian Pines, University of Pavia, and Salinas. We first compare CAD-GCN with other state-of-the-art methods, where four metrics including per-class accuracy, overall accuracy (OA), average accuracy (AA), and kappa coefficient, are used to evaluate the model performance. Then we study the influence of the number of labeled pixels on the classification performance. After that, we investigate the impact of hyperparameters incorporated by the proposed CAD-GCN. Finally, we present the ablation study and also investigate the running time of our model.

\section{A. Datasets}

The performance of our proposed CAD-GCN is evaluated on three real-world benchmark datasets, i.e., the Indian Pines ${ }^{1}$

\footnotetext{
${ }^{1}$ http://www.ehu.eus/ccwintco/index.php?title=Hyperspectral_Remote_Sen sing_Scenes\#Indian_Pines
} 


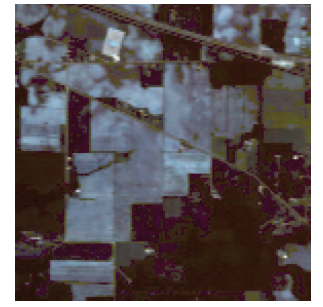

(a)

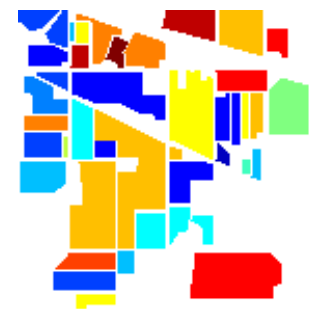

(b)
Alfal

$\square$ Corn-notill Corn-mintill Corn Grass-pasture Grass-trees

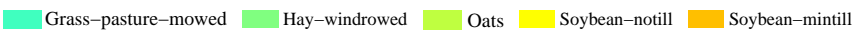
Soybean-clean Wheat $\square$ Woods $\square$ Buildings-grass-trees-drives Stone-steel-towers

Fig. 3. Indian Pines. (a) False color image. (b) Ground truth map.

TABLE I

Numbers of Labeled AND UNLABEled PiXels of All Classes in INDIAN PINES DATASET

\begin{tabular}{cccc}
\hline ID & Class & \#Labeled & \#Unlabeled \\
\hline 1 & Alfalfa & 30 & 16 \\
2 & Corn-notill & 30 & 1398 \\
3 & Corn-mintill & 30 & 800 \\
4 & Corn & 30 & 207 \\
5 & Grass-pasture & 30 & 453 \\
6 & Grass-trees & 30 & 700 \\
7 & Grass-pasture-mowed & 15 & 13 \\
8 & Hay-windrowed & 30 & 448 \\
9 & Oats & 15 & 5 \\
10 & Soybean-notill & 30 & 942 \\
11 & Soybean-mintill & 30 & 2425 \\
12 & Soybean-clean & 30 & 563 \\
13 & Wheat & 30 & 175 \\
14 & Woods & 30 & 1235 \\
15 & Buildings-grass-trees-drives & 30 & 356 \\
16 & Stone-steel-towers & 30 & 63 \\
\hline
\end{tabular}

the University of Pavi ${ }^{2}$, and the Salinas ${ }^{3}$, which will be introduced below.

1) Indian Pines: The Indian Pines dataset was gathered by Airborne Visible/Infrared Imaging Spectrometer sensor in 1992, which records north-western India. This dataset consists of $145 \times 145$ pixels with a spatial resolution of $20 \mathrm{~m} \times 20 \mathrm{~m}$, and there are 220 spectral channels covering the range from $0.4 \mu \mathrm{m}$ to $2.5 \mu \mathrm{m}$. As a usual step, 20 water absorption and noisy bands are removed, and the remaining 200 bands are retained. The original ground truth of the Indian Pines dataset includes 16 land-cover classes, such as 'Alfalfa', 'Corn-notill', 'Corn-mintill', etc. Fig. 3 exhibits the false color image and ground truth map of the Indian Pines dataset. The amounts of labeled and unlabeled pixels of various classes are listed in Table [

2) University of Pavia: The University of Pavia dataset captures the Pavia University of Italy with the ROSIS sensor. This dataset consists of $610 \times 340$ pixels with a spatial reso-

\footnotetext{
${ }^{2}$ http://www.ehu.eus/ccwintco/index.php?title=Hyperspectral_Remote_Sen sing_Scenes\#Pavia_University_scene

${ }^{3}$ http://www.ehu.eus/ccwintco/index.php?title=Hyperspectral_Remote_Sen sing_Scenes\#Salinas_scene
}

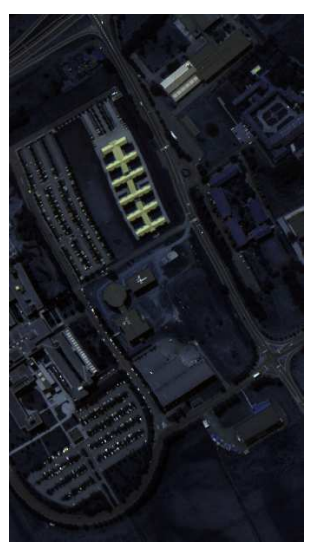

(a)

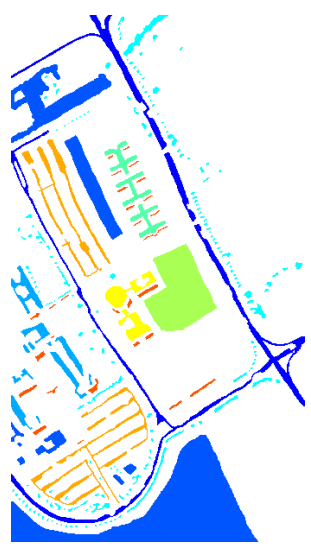

(b)
Asphalt $\quad$ Meadows $\square$ Gravel $\square$ Trees $\square$ Painted metal sheets $\square$ Bare soil Bitumen Self-blocking bricks $\quad$ Shadows

Fig. 4. University of Pavia. (a) False color image. (b) Ground truth map.

TABLE II

Numbers of LABeled and UNLABEled PiXels of All Classes in UNIVERSITY OF PAVIA DATASET

\begin{tabular}{cccc}
\hline ID & Class & \#Labeled & \#Unlabeled \\
\hline 1 & Asphalt & 30 & 6601 \\
2 & Meadows & 30 & 18619 \\
3 & Gravel & 30 & 2069 \\
4 & Trees & 30 & 3034 \\
5 & Painted metal sheets & 30 & 1315 \\
6 & Bare soil & 30 & 4999 \\
7 & Bitumen & 30 & 1300 \\
8 & Self-blocking bricks & 30 & 3652 \\
9 & Shadows & 30 & 917 \\
\hline
\end{tabular}

lution of $1.3 \mathrm{~m} \times 1.3 \mathrm{~m}$ and has 103 spectral channels in the wavelength ranging from $0.43 \mu \mathrm{m}$ to $0.86 \mu \mathrm{m}$ after removing noisy bands. The University of Pavia dataset includes 9 landcover classes, such as 'Asphalt', 'Meadows', 'Gravel', etc., which are displayed in Fig. 4 . Table II] shows the amounts of labeled and unlabeled pixels of each class.

3) Salinas: The Salinas dataset is another classic HSI which is collected by the AVIRIS sensor over Salinas Valley, California. This dataset comprises 204 spectral bands (20 water absorption bands are removed) and $512 \times 217$ pixels with a spatial resolution of $3.7 \mathrm{~m}$. The Salinas dataset contains 16 land-cover classes, such as 'Fallow', 'Stubble', 'Celery', and so on. Fig. 5 exhibits the false color image and ground truth map of the Salinas dataset. The numbers of labeled and unlabeled pixels of different classes are listed in Table III

\section{B. Experimental Settings}

In our experiments, the proposed CAD-GCN algorithm is implemented by TensorFlow with Adam optimizer. For all the adopted three datasets mentioned in Section IV-A, usually 30 labeled pixels (i.e., examples) per class are randomly chosen for training, and 15 labeled pixels are chosen if the corresponding class contains less than 30 pixels. During training, $90 \%$ of the labeled examples are used to learn the network 

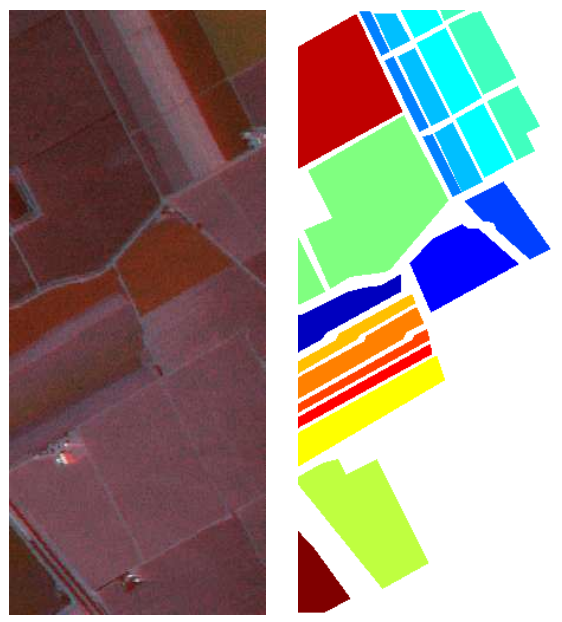

(a)

(b)

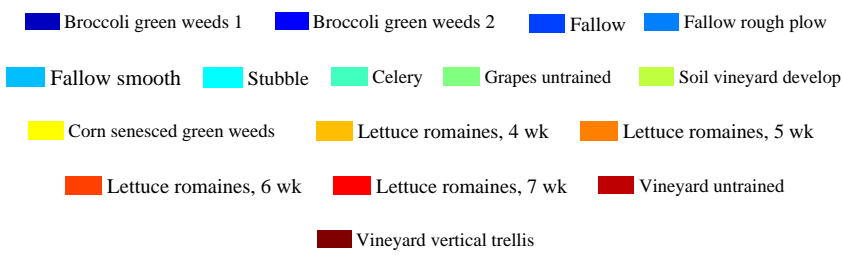

Fig. 5. Salinas. (a) False color image. (b) Ground truth map.

TABLE III

Numbers of LABElEd AND UnLABEled PiXels of All Classes iN SALINAS DATASET

\begin{tabular}{cccc}
\hline ID & Class & \#Labeled & \#Unlabeled \\
\hline 1 & Broccoli green weeds 1 & 30 & 1979 \\
2 & Broccoli green weeds 2 & 30 & 3696 \\
3 & Fallow & 30 & 1946 \\
4 & Fallow rough plow & 30 & 1364 \\
5 & Fallow smooth & 30 & 2648 \\
6 & Stubble & 30 & 3929 \\
7 & Celery & 30 & 3549 \\
8 & Grapes untrained & 30 & 11241 \\
9 & Soil vineyard develop & 30 & 6173 \\
10 & Corn senesced green weeds & 30 & 3248 \\
11 & Lettuce romaines, 4 wk & 30 & 1038 \\
12 & Lettuce romaines, 5 wk & 30 & 1897 \\
13 & Lettuce romaines, 6 wk & 30 & 886 \\
14 & Lettuce romaines, 7 wk & 30 & 1040 \\
15 & Vineyard untrained & 30 & 7238 \\
16 & Vineyard vertical trellis & 30 & 1777 \\
\hline
\end{tabular}

parameters and $10 \%$ are used as validation set to tune the hyperparameters. Meanwhile, all the unlabeled examples are used as the test set to evaluate the classification performance.

To evaluate the classification ability of our proposed CADGCN, other recent state-of-the-art HSI classification methods are also utilized for comparison. Specifically, we employ two GCN-based methods, i.e., Graph Convolutional Network (GCN) [17] and Spectral-Spatial Graph Convolutional Network $\left(\mathrm{S}^{2} \mathrm{GCN}\right)$ [47], together with two CNN-based methods, i.e., Recurrent 2D-CNN (R-2D-CNN) [51] and CNN-PixelPair Features (CNN-PPF) [52]. Meanwhile, we also compare the proposed CAD-GCN with two traditional HSI classification methods, namely Joint collaborative representation and
SVM with Decision Fusion (JSDF) [54] and Multiple Feature Learning (MFL) [53], respectively. All these methods are implemented ten times with different labeled pixels on each hyperspectral dataset, and the mean accuracies together with the standard deviations over these ten independent implementations are reported.

\section{Classification Results}

To show the effectiveness of our proposed CAD-GCN, here we quantitatively and qualitatively evaluate the classification performance by comparing CAD-GCN with the aforementioned baseline methods.

1) Results on the Indian Pines Dataset: The quantitative results acquired by different methods on the Indian Pines dataset are presented in Table IV, and the highest record regarding each class (i.e., each row) has been highlighted in bold. As shown by Table IV, our proposed CAD-GCN achieves the top level performance among all the methods in terms of OA, AA, and Kappa coefficient, and the standard deviations are very small as well. Meanwhile, the proposed CAD-GCN acquires stable and very high classification accuracies on most of the land-cover classes. All these statistics demonstrate the effectiveness of our CAD-GCN in HSI classification.

The classification maps generated by different methods on the Indian Pines dataset are exhibited in Fig. 6. To facilitate the comparison among the investigated methods, the ground truth map is also provided in Fig. 6(a). A visual inspection reveals that the proposed CAD-GCN method produces much more compact classification map and shows fewer misclassifications than other methods. More concretely, in the classification maps of GCN, S ${ }^{2}$ GCN, R-2D-CNN, CNN-PPF, and MFL, the errors are almost uniformly distributed (the salt-and-pepper effect in the homogeneous regions), while in the classification maps of JSDF and our proposed CAD-GCN, the errors only appear in some highly heterogeneous areas, where the spatial separability between classes is quite low. For instance, in Figs. 6(b) 6(f), the middle and the bottom left parts of the classification maps which correspond to 'Soybean-mintill' are highly confusing. Moreover, by comparing CAD-GCN with JSDF, we can also find that JSDF produces more errors around class boundaries than our CAD-GCN method, which reveals the good discriminability of the proposed CAD-GCN in boundary regions.

2) Results on the University of Pavia Dataset: In Table V different methods are compared on the aforementioned three datasets, where per-class accuracy, OA, AA, and Kappa coefficient are reported, and the highest value in each row is highlighted in bold. From Table V, we can conclude that our proposed CAD-GCN method achieves the best result in terms of OA and Kappa coefficient among all the competitors. Compared with the two CNN-based methods (i.e., R-2D-CNN and CNN-PPF), the proposed CAD-GCN increases the OA by $9.94 \%$ and $3.60 \%$, respectively, which suggests that the refined contextual relations captured by our CAD-GCN is superior to the spatial context characterized by the fixed convolutional kernels of CNN.

Fig. 7 visualizes the classification results generated by the seven different methods on the University of Pavia dataset. As 
TABLE IV

Per-Class Accuracy, OA, AA (\%), and Kappa Coefficient Achieved by Different Methods on Indian Pines Dataset

\begin{tabular}{cccccccc}
\hline ID & GCN [17] & $S^{2}$ GCN [4] & R-2D-CNN [51] & CNN-PPF [52] & MFL [53] & JSDF [54 $]$ & CAD-GCN \\
\hline 1 & $95.00 \pm 2.80$ & $\mathbf{1 0 0 . 0 0} \pm \mathbf{0 . 0 0}$ & $\mathbf{1 0 0 . 0 0} \pm \mathbf{0 . 0 0}$ & $95.00 \pm 2.64$ & $97.64 \pm 0.88$ & $\mathbf{1 0 0 . 0 0} \pm \mathbf{0 . 0 0}$ & $\mathbf{1 0 0 . 0 0} \pm \mathbf{0 . 0 0}$ \\
2 & $56.71 \pm 4.42$ & $84.43 \pm 2.50$ & $54.94 \pm 2.23$ & $73.53 \pm 5.61$ & $67.93 \pm 0.42$ & $\mathbf{9 0 . 7 5} \pm \mathbf{3 . 1 9}$ & $89.24 \pm 4.11$ \\
3 & $51.50 \pm 2.56$ & $82.87 \pm 5.53$ & $73.31 \pm 4.33$ & $81.34 \pm 3.76$ & $71.03 \pm 0.63$ & $77.84 \pm 3.81$ & $\mathbf{9 4 . 2 0} \pm \mathbf{3 . 2 6}$ \\
4 & $84.64 \pm 3.16$ & $93.08 \pm 1.95$ & $84.06 \pm 12.98$ & $91.84 \pm 3.53$ & $85.84 \pm 0.70$ & $\mathbf{9 9 . 8 6} \pm \mathbf{0 . 3 3}$ & $98.52 \pm 1.15$ \\
5 & $83.71 \pm 3.20$ & $\mathbf{9 7 . 1 3} \pm \mathbf{1 . 3 4}$ & $87.64 \pm 0.31$ & $93.69 \pm 0.84$ & $89.36 \pm 0.48$ & $87.20 \pm 2.73$ & $95.12 \pm 1.89$ \\
6 & $94.03 \pm 2.11$ & $97.29 \pm 1.27$ & $91.21 \pm 4.34$ & $97.46 \pm 1.01$ & $97.66 \pm 0.27$ & $\mathbf{9 8 . 5 4} \pm \mathbf{0 . 2 8}$ & $95.42 \pm 5.02$ \\
7 & $92.31 \pm 0.00$ & $92.31 \pm 0.00$ & $\mathbf{1 0 0 . 0 0} \pm \mathbf{0 . 0 0}$ & $75.38 \pm 8.73$ & $95.06 \pm 0.79$ & $\mathbf{1 0 0 . 0 0} \pm \mathbf{0 . 0 0}$ & $98.65 \pm 2.68$ \\
8 & $96.61 \pm 1.86$ & $99.03 \pm 0.93$ & $99.11 \pm 0.95$ & $98.01 \pm 0.69$ & $99.62 \pm 0.05$ & $\mathbf{9 9 . 8 0} \pm \mathbf{0 . 3 1}$ & $99.68 \pm 0.54$ \\
9 & $\mathbf{1 0 0 . 0 0} \pm \mathbf{0 . 0 0}$ & $\mathbf{1 0 0 . 0 0} \pm \mathbf{0 . 0 0}$ & $\mathbf{1 0 0 . 0 0} \pm \mathbf{0 . 0 0}$ & $\mathbf{1 0 0 . 0 0} \pm \mathbf{0 . 0 0}$ & $98.00 \pm 0.94$ & $\mathbf{1 0 0 . 0 0} \pm \mathbf{0 . 0 0}$ & $\mathbf{1 0 0 . 0 0} \pm \mathbf{0 . 0 0}$ \\
10 & $77.47 \pm 1.24$ & $\mathbf{9 3 . 7 7} \pm \mathbf{3 . 7 2}$ & $70.81 \pm 5.11$ & $82.30 \pm 1.55$ & $76.41 \pm 0.64$ & $89.99 \pm 4.24$ & $89.09 \pm 4.19$ \\
11 & $56.56 \pm 1.53$ & $84.98 \pm 2.82$ & $56.35 \pm 1.08$ & $62.64 \pm 3.32$ & $73.78 \pm 0.59$ & $76.75 \pm 5.12$ & $\mathbf{9 2 . 1 8} \pm \mathbf{2 . 0 0}$ \\
12 & $58.29 \pm 6.58$ & $80.05 \pm 5.17$ & $63.06 \pm 12.81$ & $88.92 \pm 2.50$ & $70.92 \pm 0.80$ & $87.10 \pm 2.82$ & $\mathbf{9 6 . 0 3} \pm \mathbf{2 . 6 5}$ \\
13 & $\mathbf{1 0 0 . 0 0} \pm \mathbf{0 . 0 0}$ & $99.43 \pm 0.00$ & $98.86 \pm 1.62$ & $98.80 \pm 0.57$ & $98.80 \pm 0.08$ & $99.89 \pm 0.36$ & $99.75 \pm 0.29$ \\
14 & $80.03 \pm 3.93$ & $96.73 \pm 0.92$ & $88.74 \pm 2.58$ & $86.49 \pm 2.23$ & $90.12 \pm 0.53$ & $97.21 \pm 2.78$ & $\mathbf{9 9 . 3 6} \pm \mathbf{0 . 6 9}$ \\
15 & $69.55 \pm 6.66$ & $86.80 \pm 3.42$ & $87.08 \pm 2.78$ & $86.71 \pm 4.36$ & $96.05 \pm 0.35$ & $\mathbf{9 9 . 5 8} \pm \mathbf{0 . 6 8}$ & $99.04 \pm 1.12$ \\
16 & $98.41 \pm 0.00$ & $\mathbf{1 0 0 . 0 0} \pm \mathbf{0 . 0 0}$ & $97.62 \pm 1.12$ & $92.70 \pm 3.45$ & $97.54 \pm 0.23$ & $\mathbf{1 0 0 . 0 0} \pm \mathbf{0 . 0 0}$ & $98.18 \pm 3.89$ \\
\hline OA & $69.24 \pm 1.56$ & $89.49 \pm 1.08$ & $72.11 \pm 1.28$ & $80.09 \pm 1.56$ & $80.22 \pm 0.20$ & $88.34 \pm 1.39$ & $\mathbf{9 4 . 0 6} \pm \mathbf{0 . 8 5}$ \\
AA & $80.93 \pm 1.71$ & $92.99 \pm 1.04$ & $84.55 \pm 1.79$ & $87.80 \pm 1.53$ & $87.85 \pm 0.19$ & $94.03 \pm 0.55$ & $\mathbf{9 6 . 5 3} \pm \mathbf{0 . 5 5}$ \\
Kappa & $65.27 \pm 1.80$ & $88.00 \pm 1.23$ & $68.66 \pm 1.46$ & $77.52 \pm 1.74$ & $77.59 \pm 0.22$ & $86.80 \pm 1.55$ & $\mathbf{9 3 . 2 2} \pm \mathbf{0 . 9 7}$ \\
\hline
\end{tabular}

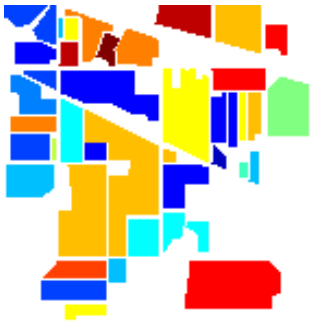

(a)

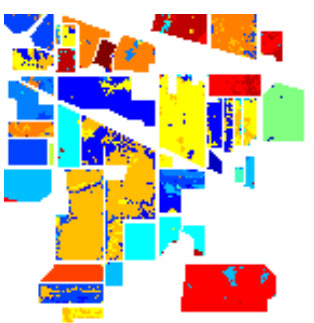

(e)

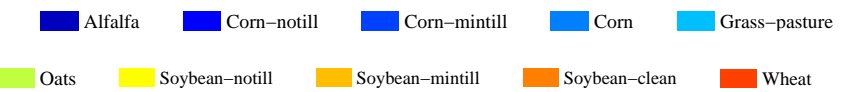

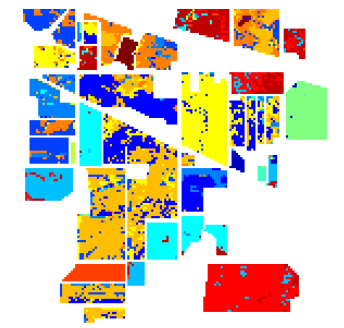

(b)

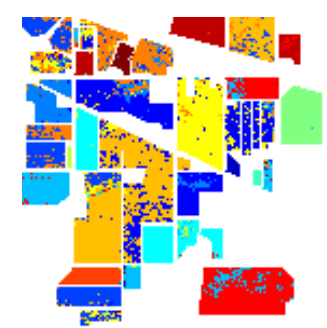

(f)

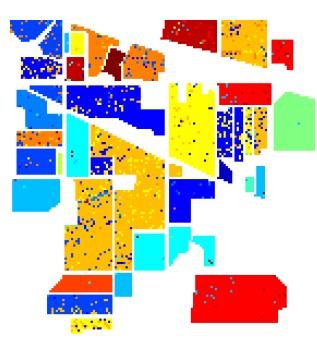

(c)

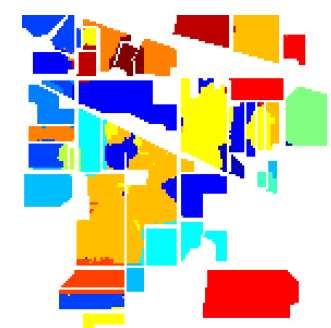

(g)

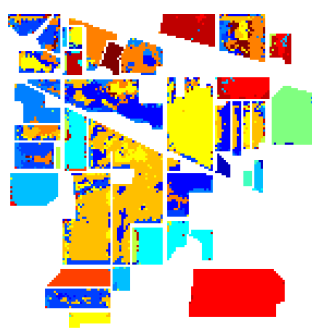

(d)

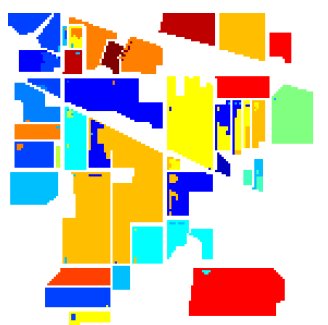

(h)

Grass-trees Grass-pasture-mowed Hay-windrowed

Woods Buildings-grass-trees-drives Stone-steel-towers

Fig. 6. Classification maps obtained by different methods on Indian Pines dataset. (a) Ground truth map; (b) GCN; (c) S ${ }^{2}$ GCN; (d) R-2D-CNN; (e) CNN-PPF; (f) MFL; (g) JSDF; (h) CAD-GCN.

depicted in Fig. 7(h), the classification map of our proposed CAD-GCN are noticeably closer to the ground truth map (see Fig. 7(a) than other methods, which is consistent with previous results in Table $\mathrm{V}$. Although GCN and $\mathrm{S}^{2} \mathrm{GCN}$ are able to capture the relations among graph nodes, they are not originally designed for accurately encoding the contextual relations of HSI. Different from GCN and $\mathrm{S}^{2} \mathrm{GCN}$, our CADGCN employs graph projection and dynamic graph refinement operations to effectively exploit the improved contextual relations of HSI. As a result, GCN and $\mathrm{S}^{2} \mathrm{GCN}$ which use the fixed coarse graph convolution produce more errors than our proposed CAD-GCN.
3) Results on the Salinas Dataset: Table VI presents the experimental results of different methods on the Salinas dataset. The proposed CAD-GCN is obviously superior to the CNNbased methods (i.e., R-2D-CNN and CNN-PPF) and all the other competitors. For instance, in Table VI CAD-GCN yields over $10 \%$ higher OA than R-2D-CNN and approximately $8 \%$ higher OA than CNN-PPF. Especially in some classes such as 'Grapes untrained' (ID $=8$ ) and 'Vineyard untrained' $(\mathrm{ID}=15)$, the class-specific accuracies of our proposed CADGCN are even approximately $20 \%$ higher than those of the CNN-based methods.

Fig. 8 provides a visual comparison of the classification 
TABLE V

Per-Class Accuracy, OA, AA (\%), and Kappa Coefficient Achieved by Different Methods on University of Pavia Dataset

\begin{tabular}{cccccccc}
\hline ID & GCN [17 & S $^{2}$ GCN [47] & R-2D-CNN [51] & CNN-PPF [52] & MFL [53] & JSDF [54 $]$ & CAD-GCN \\
\hline 1 & $69.78 \pm 4.71$ & $92.87 \pm 3.79$ & $84.96 \pm 0.56$ & $\mathbf{9 5 . 7 3} \pm \mathbf{0 . 8 0}$ & $92.46 \pm 0.27$ & $82.40 \pm 4.07$ & $83.17 \pm 3.61$ \\
2 & $54.10 \pm 10.54$ & $87.06 \pm 4.47$ & $79.99 \pm 2.29$ & $84.01 \pm 1.99$ & $87.22 \pm 0.56$ & $90.76 \pm 3.74$ & $\mathbf{9 5 . 4 9} \pm \mathbf{2 . 1 3}$ \\
3 & $69.69 \pm 4.48$ & $87.97 \pm 4.77$ & $89.49 \pm 0.17$ & $86.45 \pm 1.94$ & $81.59 \pm 0.74$ & $86.71 \pm 4.14$ & $\mathbf{9 7 . 0 4} \pm \mathbf{1 . 7 3}$ \\
4 & $91.23 \pm 7.02$ & $90.85 \pm 0.94$ & $\mathbf{9 8 . 1 2} \pm \mathbf{0 . 6 5}$ & $91.70 \pm 2.06$ & $93.69 \pm 0.42$ & $92.88 \pm 2.16$ & $78.16 \pm 5.19$ \\
5 & $98.74 \pm 0.11$ & $\mathbf{1 0 0 . 0 0} \pm \mathbf{0 . 0 0}$ & $99.85 \pm 0.11$ & $99.93 \pm 0.04$ & $99.26 \pm 0.05$ & $\mathbf{1 0 0 . 0 0} \pm \mathbf{0 . 0 0}$ & $98.01 \pm 1.34$ \\
6 & $65.34 \pm 10.53$ & $88.69 \pm 2.64$ & $76.79 \pm 7.40$ & $93.57 \pm 1.28$ & $90.38 \pm 0.58$ & $94.30 \pm 4.55$ & $\mathbf{9 6 . 7 0} \pm \mathbf{1 . 3 4}$ \\
7 & $86.64 \pm 4.68$ & $98.88 \pm 1.08$ & $88.69 \pm 4.57$ & $93.53 \pm 0.72$ & $\mathbf{9 9 . 4 0} \pm \mathbf{0 . 0 5}$ & $96.62 \pm 1.37$ & $99.05 \pm 1.25$ \\
8 & $72.26 \pm 2.63$ & $89.97 \pm 3.28$ & $67.54 \pm 5.67$ & $83.83 \pm 1.60$ & $81.74 \pm 0.55$ & $\mathbf{9 4 . 6 9} \pm \mathbf{3 . 7 4}$ & $93.83 \pm 3.23$ \\
9 & $\mathbf{9 9 . 9 3} \pm \mathbf{0 . 0 6}$ & $98.89 \pm 0.53$ & $99.84 \pm 0.08$ & $99.47 \pm 0.34$ & $99.80 \pm 0.03$ & $99.56 \pm 0.36$ & $82.66 \pm 4.54$ \\
\hline OA & $66.19 \pm 3.43$ & $89.74 \pm 1.70$ & $82.38 \pm 0.88$ & $88.72 \pm 0.95$ & $89.14 \pm 0.26$ & $90.82 \pm 1.30$ & $\mathbf{9 2 . 3 2} \pm \mathbf{0 . 9 8}$ \\
AA & $78.63 \pm 1.23$ & $92.80 \pm 0.47$ & $87.25 \pm 0.68$ & $92.02 \pm 0.37$ & $91.73 \pm 0.14$ & $\mathbf{9 3 . 1 0} \pm \mathbf{0 . 6 5}$ & $91.57 \pm 0.71$ \\
Kappa & $58.39 \pm 3.28$ & $86.65 \pm 2.06$ & $77.31 \pm 0.97$ & $85.43 \pm 1.18$ & $85.91 \pm 0.32$ & $88.02 \pm 1.62$ & $\mathbf{8 9 . 9 1} \pm \mathbf{1 . 2 5}$ \\
\hline
\end{tabular}

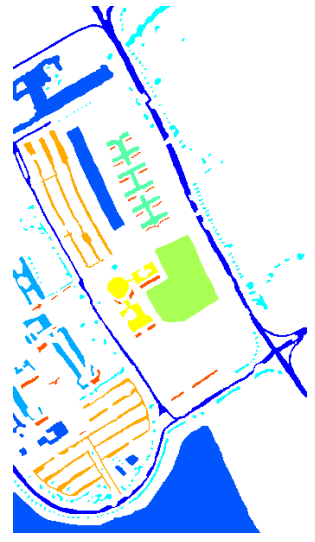

(a)

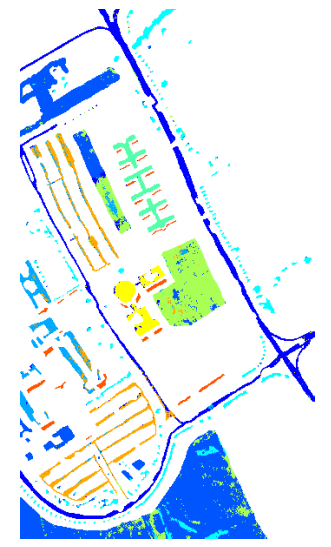

(e)

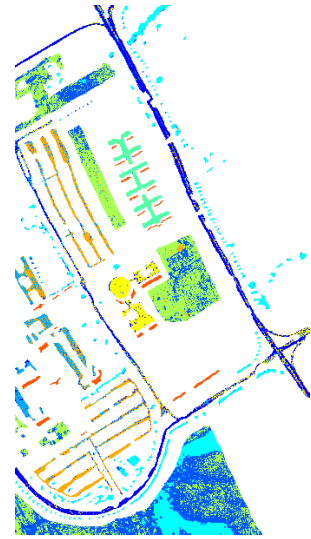

(b)

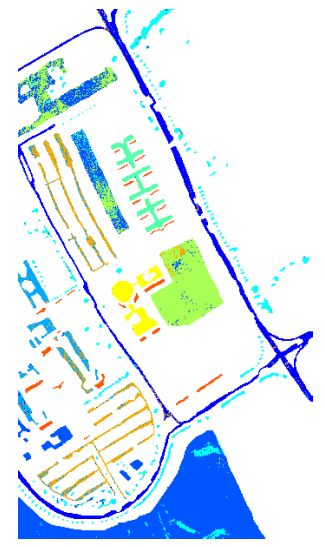

(f)

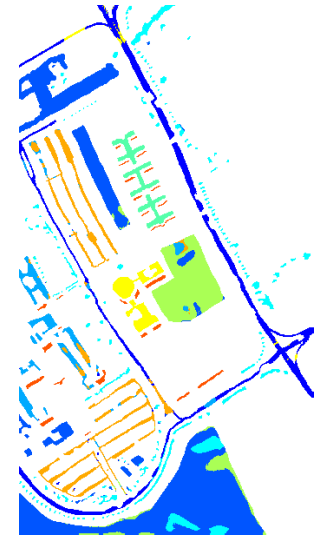

(c)

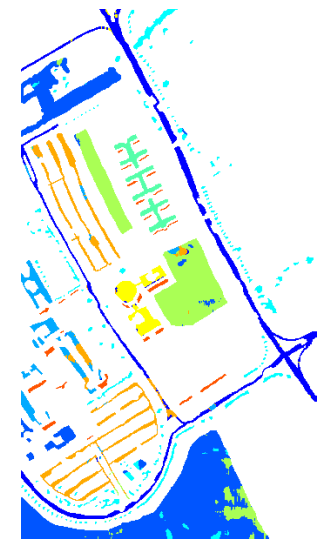

(g)

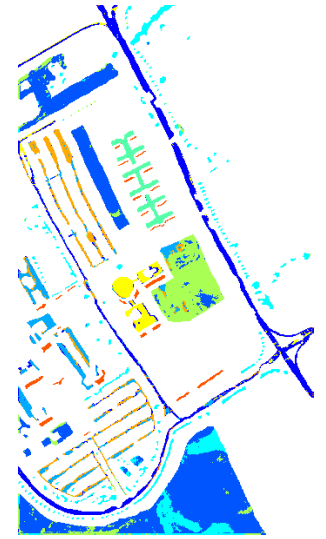

(d)

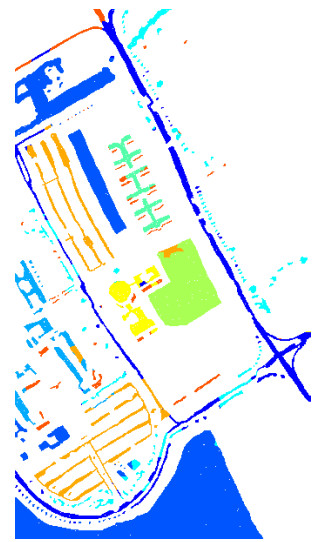

(h)

Asphalt

Meadows

Gravel

Trees

Painted metal sheets

Bare soil Bitumen

Self-blocking bricks

Shadows

Fig. 7. Classification maps obtained by different methods on University of Pavia dataset. (a) Ground truth map; (b) GCN; (c) S ${ }^{2}$ GCN; (d) R-2D-CNN; (e) CNN-PPF; (f) MFL; (g) JSDF; (h) CAD-GCN.

results obtained by different methods. It is observable that some areas in the classification map of our proposed CADGCN are less noisy than those of other methods, e.g., the regions of 'Grapes untrained' and 'Vineyard untrained', which is in consistence with the results listed in Table VI.

\section{Impact of the Number of Labeled Examples}

In this experiment, the classification performances of the aforementioned seven methods with different numbers of labeled examples (i.e., pixels) for training are investigated. To be specific, we vary the number of labeled examples per class form 5 to 30 with an interval of 5, and report the OA acquired by all the methods on the Indian Pines, the University of Pavia, and the Salinas datasets (see Fig. 9p. From the results, we 
TABLE VI

Per-Class Accuracy, OA, AA (\%), and Kappa Coefficient Achieved by Different Methods on Salinas Dataset

\begin{tabular}{cccccccc}
\hline ID & GCN [17] & $\mathrm{S}^{2} \mathrm{GCN}[47]$ & R-2D-CNN [51] & CNN-PPF [52] & MFL [53] & JSDF [54] & CAD-GCN \\
\hline 1 & $98.62 \pm 0.86$ & $99.01 \pm 0.44$ & $98.17 \pm 0.34$ & $99.77 \pm 0.21$ & $98.41 \pm 0.09$ & $\mathbf{1 0 0 . 0 0} \pm \mathbf{0 . 0 0}$ & $\mathbf{1 0 0 . 0 0} \pm \mathbf{0 . 0 0}$ \\
2 & $99.07 \pm 1.21$ & $99.18 \pm 0.59$ & $97.79 \pm 1.10$ & $98.69 \pm 0.89$ & $99.04 \pm 0.06$ & $\mathbf{1 0 0 . 0 0} \pm \mathbf{0 . 0 0}$ & $\mathbf{1 0 0 . 0 0} \pm \mathbf{0 . 0 0}$ \\
3 & $97.03 \pm 1.10$ & $97.15 \pm 2.76$ & $94.56 \pm 3.84$ & $99.50 \pm 0.49$ & $99.74 \pm 0.04$ & $\mathbf{1 0 0 . 0 0} \pm \mathbf{0 . 0 0}$ & $99.96 \pm 0.09$ \\
4 & $99.28 \pm 0.49$ & $99.11 \pm 0.55$ & $96.86 \pm 1.03$ & $99.81 \pm 0.04$ & $98.43 \pm 0.14$ & $\mathbf{9 9 . 9 3} \pm \mathbf{0 . 0 9}$ & $98.12 \pm 1.62$ \\
5 & $98.58 \pm 0.79$ & $97.55 \pm 2.35$ & $97.54 \pm 1.08$ & $96.64 \pm 1.26$ & $98.53 \pm 0.02$ & $\mathbf{9 9 . 7 7} \pm \mathbf{0 . 3 1}$ & $98.05 \pm 0.93$ \\
6 & $99.58 \pm 0.30$ & $99.32 \pm 0.35$ & $98.88 \pm 0.44$ & $99.32 \pm 0.86$ & $98.97 \pm 0.11$ & $\mathbf{1 0 0 . 0 0} \pm \mathbf{0 . 0 0}$ & $99.66 \pm 0.31$ \\
7 & $99.13 \pm 0.25$ & $99.06 \pm 0.27$ & $97.98 \pm 1.12$ & $99.59 \pm 0.13$ & $99.14 \pm 0.03$ & $\mathbf{9 9 . 9 9} \pm \mathbf{0 . 0 1}$ & $99.15 \pm 1.73$ \\
8 & $67.94 \pm 8.33$ & $70.68 \pm 5.20$ & $69.76 \pm 9.72$ & $74.77 \pm 4.01$ & $69.74 \pm 0.86$ & $87.79 \pm 4.89$ & $\mathbf{9 5 . 4 0} \pm \mathbf{3 . 1 5}$ \\
9 & $98.50 \pm 0.85$ & $98.32 \pm 1.79$ & $97.34 \pm 0.57$ & $98.99 \pm 0.18$ & $98.95 \pm 0.04$ & $99.67 \pm 0.33$ & $\mathbf{1 0 0 . 0 0} \pm \mathbf{0 . 0 0}$ \\
10 & $89.64 \pm 1.57$ & $90.97 \pm 2.59$ & $88.04 \pm 4.54$ & $89.32 \pm 3.04$ & $90.66 \pm 0.29$ & $96.53 \pm 2.55$ & $\mathbf{9 8 . 1 0} \pm \mathbf{1 . 1 7}$ \\
11 & $94.80 \pm 2.98$ & $98.00 \pm 1.65$ & $93.43 \pm 1.56$ & $97.65 \pm 1.49$ & $93.85 \pm 0.28$ & $99.76 \pm 0.21$ & $\mathbf{9 9 . 8 6} \pm \mathbf{0 . 2 6}$ \\
12 & $99.71 \pm 0.08$ & $99.56 \pm 0.59$ & $96.79 \pm 0.99$ & $99.82 \pm 0.30$ & $97.85 \pm 0.31$ & $\mathbf{1 0 0 . 0 0} \pm \mathbf{0 . 0 0}$ & $97.94 \pm 0.84$ \\
13 & $97.99 \pm 0.61$ & $97.83 \pm 0.72$ & $95.54 \pm 0.81$ & $97.70 \pm 0.50$ & $99.12 \pm 0.10$ & $\mathbf{1 0 0 . 0 0} \pm \mathbf{0 . 0 0}$ & $97.96 \pm 1.01$ \\
14 & $93.58 \pm 2.60$ & $95.75 \pm 1.65$ & $92.98 \pm 1.85$ & $94.14 \pm 1.22$ & $94.52 \pm 0.32$ & $98.71 \pm 0.72$ & $\mathbf{9 9 . 1 6} \pm \mathbf{0 . 7 1}$ \\
15 & $66.18 \pm 9.08$ & $70.36 \pm 3.62$ & $74.40 \pm 7.97$ & $79.12 \pm 1.99$ & $71.09 \pm 0.83$ & $81.86 \pm 5.26$ & $\mathbf{9 7 . 5 2} \pm \mathbf{1 . 9 2}$ \\
16 & $97.24 \pm 1.21$ & $96.90 \pm 1.97$ & $91.87 \pm 1.87$ & $98.65 \pm 0.31$ & $99.37 \pm 0.05$ & $98.99 \pm 0.63$ & $\mathbf{9 9 . 7 6} \pm \mathbf{0 . 6 7}$ \\
\hline OA & $87.16 \pm 0.85$ & $88.39 \pm 1.01$ & $87.63 \pm 1.26$ & $90.52 \pm 0.77$ & $88.36 \pm 0.22$ & $94.67 \pm 0.77$ & $\mathbf{9 8 . 2 3} \pm \mathbf{0 . 5 4}$ \\
AA & $93.55 \pm 0.39$ & $94.30 \pm 0.47$ & $92.62 \pm 0.49$ & $95.22 \pm 0.34$ & $94.21 \pm 0.08$ & $97.69 \pm 0.34$ & $\mathbf{9 8 . 7 9} \pm \mathbf{0 . 2 2}$ \\
Kappa & $85.74 \pm 0.92$ & $87.10 \pm 1.12$ & $86.28 \pm 1.37$ & $89.46 \pm 0.85$ & $87.06 \pm 0.24$ & $94.06 \pm 0.85$ & $\mathbf{9 8 . 0 3} \pm \mathbf{0 . 6 0}$ \\
\hline
\end{tabular}

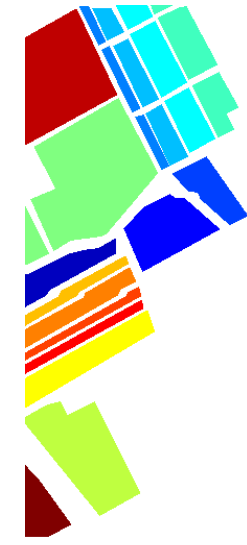

(a)

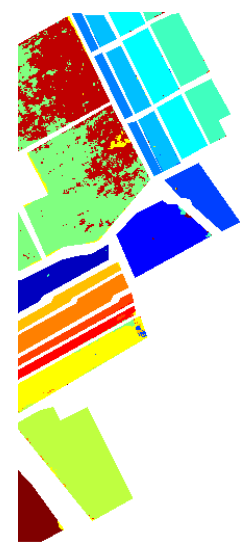

(e)

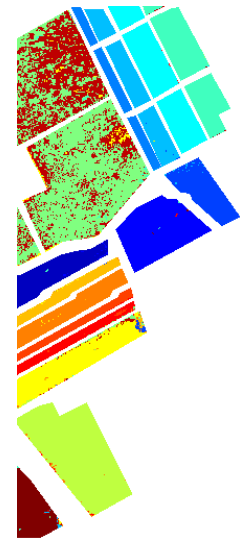

(b)

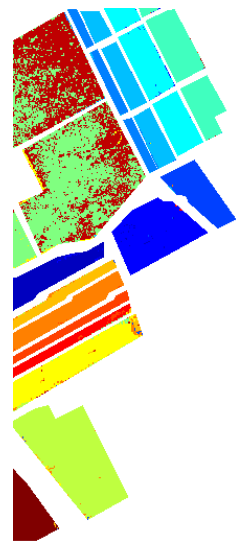

(f)

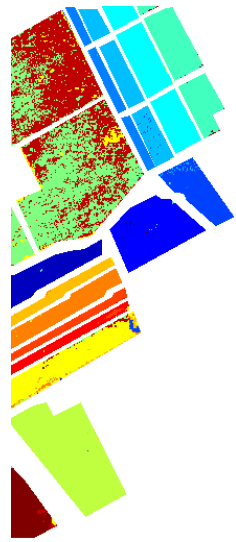

(c)

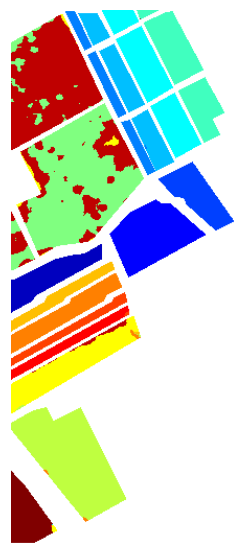

(g)

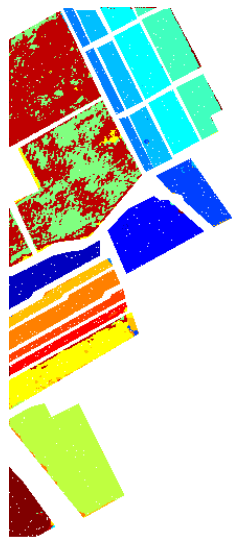

(d)

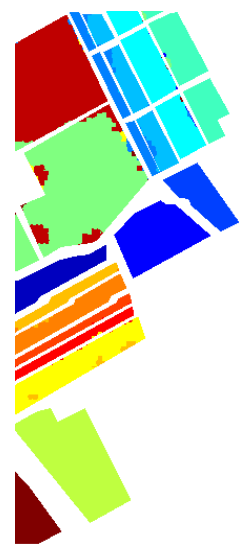

(h)

Broccoli green weeds 1

Fallow Fallow rough plow

Fallow smooth

Stubble $\quad$ Celery $\quad$ Grapes untrained $\quad$ Soil vineyard develop

Corn senesced green weeds

Lettuce romaines, $4 \mathrm{wk}$ Lettuce romaines, $5 \mathrm{wk}$

Lettuce romaines, $6 \mathrm{wk}$

Lettuce romaines, $7 \mathrm{wk}$

Vineyard untrained

Vineyard vertical trellis

Fig. 8. Classification maps obtained by different methods on Salinas dataset. (a) Ground truth map; (b) GCN; (c) S ${ }^{2}$ GCN; (d) R-2D-CNN; (e) CNN-PPF; (f) MFL; (g) JSDF; (h) CAD-GCN. 


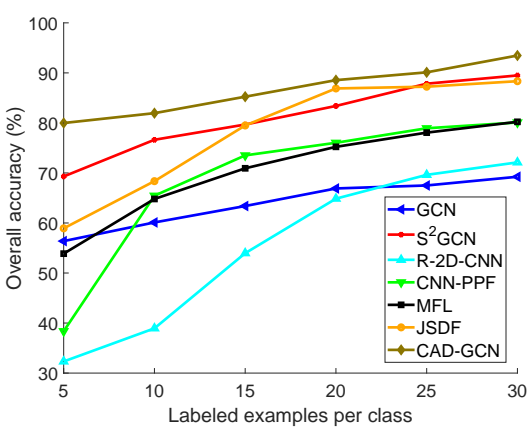

(a)

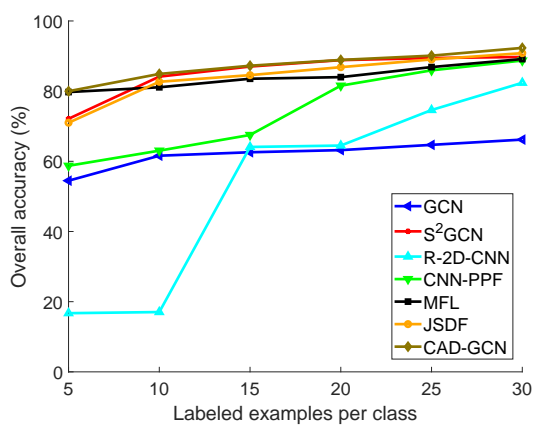

(b)

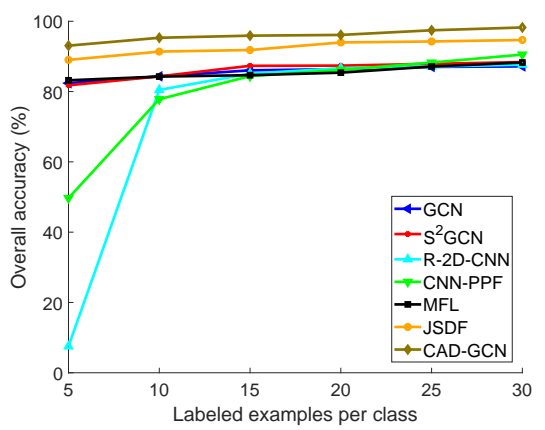

(c)

Fig. 9. Overall accuracies of various methods under different numbers of labeled examples per class. (a) Indian Pines dataset; (b) University of Pavia dataset; (c) Salinas dataset.

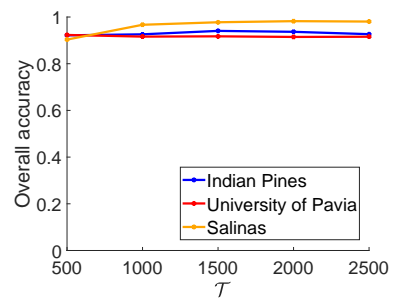

(a)

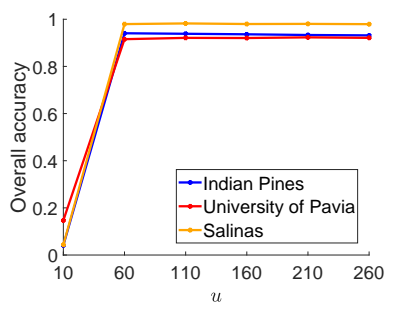

(c)

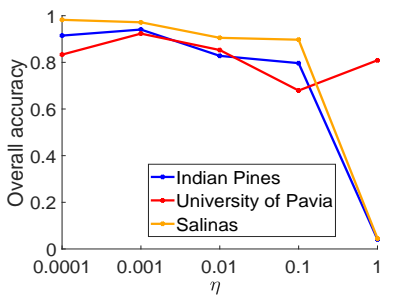

(b)

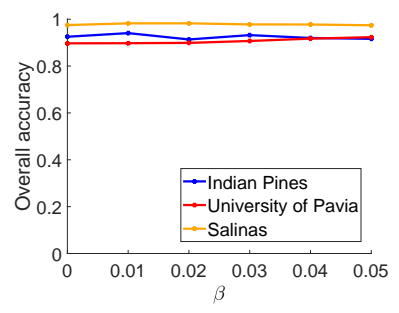

(d)

Fig. 10. Parametric sensitivity of (a) $\mathcal{T}$, (b) $\eta$, (c) $u$, and (d) $\beta$ of our proposed CAD-GCN

datasets, which verifies the effectiveness of contextual relations captured by CAD-GCN. Another interesting observation is that even if the labeled examples are quite limited (i.e., 5 or 10 labeled examples per class), our CAD-GCN still achieves relatively high $\mathrm{OA}$, which suggests good stability of CADGCN in HSI classification tasks.

\section{E. Impact of Hyperparameters}

There are several important hyperparameters that should be manually tuned in the designed CAD-GCN architecture. Here, we will evaluate in detail the sensitivity of the classification performance to different hyperparameter settings of the proposed CAD-GCN. Since GCN-based methods usually do not require deep structure to achieve excellent performance [47], [55], we empirically employ two convolutional layers for all the three datasets. The hyperparameters to be pretuned manually mainly include the number of iterations $\mathcal{T}$, the learning rate $\eta$, the number of hidden units $u$, and the threshold $\beta$ used in dynamic graph refinement. Therefore, we examine the test accuracy of CAD-GCN by varying one of $\mathcal{T}, \eta$, $u$, and

TABLE VII

Per-Class Accuracy, OA, AA (\%), and Kappa CoefFicient ACHIEved by Different Model Settings on Indian Pines Dataset

\begin{tabular}{ccccc}
\hline ID & CAD-GCN-v1 & CAD-GCN-v2 & CAD-GCN-v3 & CAD-GCN \\
\hline 1 & $98.85 \pm 2.05$ & $99.03 \pm 1.98$ & $\mathbf{1 0 0 . 0 0} \pm \mathbf{0 . 0 0}$ & $\mathbf{1 0 0 . 0 0} \pm \mathbf{0 . 0 0}$ \\
2 & $86.90 \pm 4.83$ & $86.52 \pm 4.78$ & $77.42 \pm 1.88$ & $\mathbf{8 9 . 2 4} \pm \mathbf{4 . 1 1}$ \\
3 & $92.85 \pm 4.98$ & $92.94 \pm 3.75$ & $92.28 \pm 0.60$ & $\mathbf{9 4 . 2 0} \pm \mathbf{3 . 2 6}$ \\
4 & $98.04 \pm 1.96$ & $97.92 \pm 1.95$ & $\mathbf{9 9 . 6 9} \pm \mathbf{1 . 4 0}$ & $98.52 \pm 1.15$ \\
5 & $94.63 \pm 3.22$ & $93.75 \pm 3.10$ & $93.59 \pm 1.42$ & $\mathbf{9 5 . 1 2} \pm \mathbf{1 . 8 9}$ \\
6 & $97.50 \pm 2.01$ & $\mathbf{9 7 . 8 4} \pm \mathbf{1 . 9 9}$ & $96.11 \pm 0.65$ & $95.42 \pm 5.02$ \\
7 & $96.14 \pm 5.42$ & $96.41 \pm 5.18$ & $\mathbf{1 0 0 . 0 0} \pm \mathbf{0 . 0 0}$ & $98.65 \pm 2.68$ \\
8 & $99.43 \pm 1.31$ & $98.93 \pm 1.64$ & $96.08 \pm 0.98$ & $\mathbf{9 9 . 6 8} \pm \mathbf{0 . 5 4}$ \\
9 & $\mathbf{1 0 0 . 0 0} \pm \mathbf{0 . 0 0}$ & $\mathbf{1 0 0 . 0 0} \pm \mathbf{0 . 0 0}$ & $\mathbf{1 0 0 . 0 0} \pm \mathbf{0 . 0 0}$ & $\mathbf{1 0 0 . 0 0} \pm \mathbf{0 . 0 0}$ \\
10 & $90.31 \pm 1.89$ & $89.14 \pm 3.77$ & $\mathbf{9 2 . 2 1} \pm \mathbf{0 . 8 0}$ & $89.09 \pm 4.19$ \\
11 & $89.43 \pm 3.88$ & $89.01 \pm 3.69$ & $88.14 \pm 2.13$ & $\mathbf{9 2 . 1 8} \pm \mathbf{2 . 0 0}$ \\
12 & $91.61 \pm 3.91$ & $93.59 \pm 3.27$ & $87.90 \pm 1.13$ & $\mathbf{9 6 . 0 3} \pm \mathbf{2 . 6 5}$ \\
13 & $99.83 \pm 0.32$ & $99.81 \pm 0.32$ & $\mathbf{9 9 . 8 6} \pm \mathbf{0 . 6 4}$ & $99.75 \pm 0.29$ \\
14 & $97.69 \pm 2.21$ & $98.88 \pm 2.41$ & $96.08 \pm 0.26$ & $\mathbf{9 9 . 3 6} \pm \mathbf{0 . 6 9}$ \\
15 & $98.34 \pm 2.56$ & $99.00 \pm 1.00$ & $95.32 \pm 0.33$ & $\mathbf{9 9 . 0 4} \pm \mathbf{1 . 1 2}$ \\
16 & $\mathbf{9 9 . 0 5} \pm \mathbf{1 . 1 8}$ & $98.20 \pm 2.71$ & $94.13 \pm 1.55$ & $98.18 \pm 3.89$ \\
\hline OA & $92.68 \pm 1.00$ & $92.66 \pm 1.14$ & $90.30 \pm 0.45$ & $\mathbf{9 4 . 0 6} \pm \mathbf{0 . 8 5}$ \\
AA & $95.66 \pm 0.60$ & $95.69 \pm 0.64$ & $94.30 \pm 0.22$ & $\mathbf{9 6 . 5 3} \pm \mathbf{0 . 5 5}$ \\
Kappa & $91.67 \pm 1.13$ & $91.64 \pm 1.27$ & $88.94 \pm 0.50$ & $\mathbf{9 3 . 2 2} \pm \mathbf{0 . 9 7}$ \\
\hline
\end{tabular}

$\beta$, and meanwhile fixing the remaining hyperparameters to a constant value [56]. The results on Indian Pines, University of Pavia, and Salinas datasets are shown in Fig. 10.

From the curves presented in Fig. 10, it can be observed that the aforementioned four hyperparameters are critical for the proposed CAD-GCN to achieve satisfactory performance. In order to obtain promising classification performance, we set the hyperparameters to $\mathcal{T}=1500, \eta=0.001, u=60$, $\beta=0.01$ for the Indian Pines dataset, $\mathcal{T}=500, \eta=0.001$, $u=210, \beta=0.05$ for the University of Pavia dataset, and $\mathcal{T}=2000, \eta=0.0001, u=110, \beta=0.02$ for the Salinas dataset, respectively. Note that when the threshold $\beta$ used in the edge filter (see Eq. (13)) becomes too large, the useful information contained in the graph may be filtered out. Hence, we search for the optimal $\beta$ between zero and a relatively small value 0.05 .

\section{F. Ablation Study}

As is mentioned in the introduction, the proposed CADGCN contains three critical parts for improving the contextual relations, i.e., the graph projection framework, the dynamic refinement of node similarities, and the edge filter. To shed 
TABLE VIII

Per-Class Accuracy, OA, AA (\%), and Kappa Coefficient ACHiEved by Different Model Settings on University of PaVia DATASET

\begin{tabular}{ccccc}
\hline ID & CAD-GCN-v1 & CAD-GCN-v2 & CAD-GCN-v3 & CAD-GCN \\
\hline 1 & $79.31 \pm 8.17$ & $81.64 \pm 7.14$ & $83.15 \pm 3.37$ & $\mathbf{8 3 . 1 7} \pm \mathbf{3 . 6 1}$ \\
2 & $92.09 \pm 3.89$ & $90.07 \pm 3.75$ & $93.88 \pm 3.80$ & $\mathbf{9 5 . 4 9} \pm \mathbf{2 . 1 3}$ \\
3 & $95.40 \pm 1.98$ & $95.69 \pm 3.53$ & $88.12 \pm 6.87$ & $\mathbf{9 7 . 0 4} \pm \mathbf{1 . 7 3}$ \\
4 & $76.85 \pm 5.91$ & $74.97 \pm 5.70$ & $\mathbf{8 9 . 1 4} \pm \mathbf{7 . 1 1}$ & $78.16 \pm 5.19$ \\
5 & $\mathbf{9 8 . 7 5} \pm \mathbf{0 . 7 2}$ & $98.62 \pm 1.32$ & $98.26 \pm 1.22$ & $98.01 \pm 1.34$ \\
6 & $97.10 \pm 3.96$ & $\mathbf{9 8 . 4 0} \pm \mathbf{1 . 1 8}$ & $97.52 \pm 2.10$ & $96.70 \pm 1.34$ \\
7 & $97.06 \pm 3.38$ & $97.71 \pm 3.67$ & $95.79 \pm 2.89$ & $\mathbf{9 9 . 0 5} \pm \mathbf{1 . 2 5}$ \\
8 & $92.95 \pm 3.22$ & $\mathbf{9 4 . 9 8} \pm \mathbf{3 . 0 8}$ & $85.40 \pm 4.80$ & $93.83 \pm 3.23$ \\
9 & $82.69 \pm 4.70$ & $82.96 \pm 3.90$ & $\mathbf{9 4 . 2 3} \pm \mathbf{3 . 9 0}$ & $82.66 \pm 4.54$ \\
\hline OA & $89.99 \pm 1.76$ & $89.70 \pm 1.35$ & $91.50 \pm 1.67$ & $\mathbf{9 2 . 3 2} \pm \mathbf{0 . 9 8}$ \\
AA & $90.24 \pm 1.68$ & $90.56 \pm 0.96$ & $\mathbf{9 1 . 7 2} \pm \mathbf{1 . 2 1}$ & $91.57 \pm 0.71$ \\
Kappa & $86.94 \pm 2.26$ & $86.61 \pm 1.68$ & $88.86 \pm 2.08$ & $\mathbf{8 9 . 9 1} \pm \mathbf{1 . 2 5}$ \\
\hline
\end{tabular}

TABLE IX

Per-Class Accuracy, OA, AA (\%), ANd Kappa Coefficient AChieved by Different Model Settings on Salinas Dataset

\begin{tabular}{ccccc}
\hline ID & CAD-GCN-v1 & CAD-GCN-v2 & CAD-GCN-v3 & CAD-GCN \\
\hline 1 & $\mathbf{1 0 0 . 0 0} \pm \mathbf{0 . 0 0}$ & $\mathbf{1 0 0 . 0 0} \pm \mathbf{0 . 0 0}$ & $99.58 \pm 0.75$ & $\mathbf{1 0 0 . 0 0} \pm \mathbf{0 . 0 0}$ \\
2 & $\mathbf{1 0 0 . 0 0} \pm \mathbf{0 . 0 0}$ & $\mathbf{1 0 0 . 0 0} \pm \mathbf{0 . 0 0}$ & $99.98 \pm 0.03$ & $\mathbf{1 0 0 . 0 0} \pm \mathbf{0 . 0 0}$ \\
3 & $99.98 \pm 0.06$ & $\mathbf{1 0 0 . 0 0} \pm \mathbf{0 . 0 0}$ & $99.78 \pm 0.20$ & $99.96 \pm 0.09$ \\
4 & $98.34 \pm 1.40$ & $\mathbf{9 9 . 3 3} \pm \mathbf{0 . 6 3}$ & $97.01 \pm 2.49$ & $98.12 \pm 1.62$ \\
5 & $97.03 \pm 1.64$ & $96.21 \pm 1.94$ & $96.73 \pm 1.78$ & $\mathbf{9 8 . 0 5} \pm \mathbf{0 . 9 3}$ \\
6 & $99.59 \pm 0.35$ & $99.52 \pm 0.37$ & $98.99 \pm 0.59$ & $\mathbf{9 9 . 6 6} \pm \mathbf{0 . 3 1}$ \\
7 & $99.37 \pm 1.49$ & $98.82 \pm 1.83$ & $\mathbf{9 9 . 5 4} \pm \mathbf{0 . 4 0}$ & $99.15 \pm 1.73$ \\
8 & $94.39 \pm 2.61$ & $95.17 \pm 3.67$ & $88.17 \pm 2.24$ & $\mathbf{9 5 . 4 0} \pm \mathbf{3 . 1 5}$ \\
9 & $\mathbf{1 0 0 . 0 0} \pm \mathbf{0 . 0 0}$ & $\mathbf{1 0 0 . 0 0} \pm \mathbf{0 . 0 0}$ & $99.57 \pm 0.82$ & $\mathbf{1 0 0 . 0 0} \pm \mathbf{0 . 0 0}$ \\
10 & $97.01 \pm 1.97$ & $96.96 \pm 2.58$ & $94.61 \pm 3.01$ & $\mathbf{9 8 . 1 0} \pm \mathbf{1 . 1 7}$ \\
11 & $99.77 \pm 0.51$ & $99.16 \pm 0.90$ & $96.74 \pm 2.55$ & $\mathbf{9 9 . 8 6} \pm \mathbf{0 . 2 6}$ \\
12 & $97.52 \pm 0.72$ & $97.68 \pm 1.24$ & $\mathbf{9 9 . 1 6} \pm \mathbf{0 . 7 9}$ & $97.94 \pm 0.84$ \\
13 & $97.37 \pm 1.32$ & $\mathbf{9 8 . 0 1} \pm \mathbf{0 . 4 5}$ & $97.90 \pm 1.11$ & $97.96 \pm 1.01$ \\
14 & $98.89 \pm 2.38$ & $\mathbf{9 9 . 3 4} \pm \mathbf{0 . 2 7}$ & $96.99 \pm 2.15$ & $99.16 \pm 0.71$ \\
15 & $\mathbf{9 7 . 6 7} \pm \mathbf{1 . 2 7}$ & $97.16 \pm 1.74$ & $91.14 \pm 2.23$ & $97.52 \pm 1.92$ \\
16 & $99.66 \pm 1.01$ & $99.71 \pm 0.89$ & $98.95 \pm 1.33$ & $\mathbf{9 9 . 7 6} \pm \mathbf{0 . 6 7}$ \\
\hline OA & $97.90 \pm 0.53$ & $97.95 \pm 0.71$ & $95.36 \pm 0.55$ & $\mathbf{9 8 . 2 3} \pm \mathbf{0 . 5 4}$ \\
AA & $98.54 \pm 0.30$ & $98.57 \pm 0.32$ & $97.18 \pm 0.39$ & $\mathbf{9 8 . 7 9} \pm \mathbf{0 . 2 2}$ \\
Kappa & $97.66 \pm 0.59$ & $97.72 \pm 0.79$ & $94.84 \pm 0.61$ & $\mathbf{9 8 . 0 3} \pm \mathbf{0 . 6 0}$ \\
\hline
\end{tabular}

light on the contributions of these three components, every time we report the classification results of CAD-GCN without one of the three components on the three adopted datasets (namely, the Indian Pines, the University of Pavia, and the Salinas). For simplicity, we adopt 'CAD-GCN-v1', 'CADGCN-v2', and 'CAD-GCN-v3' to represent the reduced model by removing dynamic refinement of node similarities, the edge filter, and the graph projection framework, respectively. Table VII Table VIII] and Table IX exhibit the comparative results on the aforementioned datasets. It can be obviously observed that lacking any one of the components will inevitably hurt the OA. Therefore, the graph projection framework, the dynamic refinement of node similarities, and the edge filter work collaboratively to render satisfactory classification performance.

\section{G. Running Time}

In order to reveal the advantage of our proposed CAD-GCN to the baselines in terms of efficiency, in Table $\mathrm{X}$, we report the running time of different deep models, including GCN, $\mathrm{S}^{2} \mathrm{GCN}, \mathrm{R}-2 \mathrm{D}-\mathrm{CNN}, \mathrm{CNN}-\mathrm{PPF}$, and the proposed CAD-GCN on three datasets (i.e., the Indian Pines, the University of Pavia, and the Salinas), where the number of labeled pixels
TABLE $X$

RunNing Time Comparison (In SECONDS) of DifFERENT Methods 'IP' Denotes Indian Pines Dataset and 'PaviaU' Denotes UNIVERSITY OF PAVIA DATASET

\begin{tabular}{cccccc}
\hline Dataset & GCN [17] & S $^{2}$ GCN [4] & R-2D-CNN [51] & CNN-PPF [52] & CAD-GCN \\
\hline IP & 58 & 71 & 2156 & 1495 & 44 \\
paviaU & 1783 & 1803 & 2272 & 1545 & 90 \\
Salinas & 3497 & 3528 & 2361 & 1769 & 556 \\
\hline
\end{tabular}

per class is kept identical to the experiments presented in Section IV-C The codes for all methods are written in Python, and the running time is reported on a server with a 3.60$\mathrm{GHz}$ Intel Xeon CPU with $264 \mathrm{~GB}$ of RAM and a Tesla P40 GPU. Compared with the running time of GCN, $S^{2} \mathrm{GCN}$, our proposed CAD-GCN shows remarkably higher efficiency in large-scale datasets (i.e., the University of Pavia and the Salinas dataset), which is owing much to the employment of graph projection operation. Since the graph size can be significantly reduced by graph projection, our proposed CADGCN exhibits high efficiency on all the three datasets. The comparison results demonstrate that our proposed method is effective and efficient for HSI classification.

\section{CONCLUSiON}

In this paper, we have developed a novel Context-Aware Dynamic Graph Convolutional Network (CAD-GCN) for HSI classification. In order to capture long range contextual relations, we move beyond regular image grids by learning the pixel-to-region assignment, and further encode the contextual relations among regions, so that the regions which are originally far away in the 2D space can be connected by successive graph convolutions. Moreover, we enable the node similarities and connective relationships to be dynamically updated via learning the improved distance metric and the edge filter. Therefore, the contextual relations among pixels can be gradually refined along with graph convolution, which significantly improves the performance of CAD-GCN on representation and classification of HSI. The experimental results on three realworld HSI datasets indicate that the proposed CAD-GCN is able to yield better performance when compared with the stateof-the-art HSI classification methods.

\section{REFERENCES}

[1] J. C. Harsanyi and C.-I. Chang, "Hyperspectral image classification and dimensionality reduction: an orthogonal subspace projection approach," IEEE Trans. Geosci. Remote Sens., vol. 32, no. 4, pp. 779-785, Jul. 1994.

[2] M. Zhang, W. Li, and Q. Du, "Diverse region-based CNN for hyperspectral image classification," IEEE Trans. Image Process., vol. 27, no. 6, pp. 2623-2634, Jun. 2018.

[3] F. Melgani and L. Bruzzone, "Classification of hyperspectral remote sensing images with support vector machines," IEEE Trans. Geosci. Remote Sens., vol. 42, no. 8, pp. 1778-1790, Aug. 2004.

[4] Y. Chen, N. M. Nasrabadi, and T. D. Tran, "Hyperspectral image classification using dictionary-based sparse representation," IEEE Trans. Geosci. Remote Sens., vol. 49, no. 10, pp. 3973-3985, Oct. 2011.

[5] L. He, J. Li, C. Liu, and S. Li, "Recent advances on spectral-spatial hyperspectral image classification: An overview and new guidelines," IEEE Trans. Geosci. Remote Sens., vol. 56, no. 3, pp. 1579-1597, Mar. 2018. 
[6] M. Fauvel, Y. Tarabalka, J. A. Benediktsson, J. Chanussot, and J. C. Tilton, "Advances in spectral-spatial classification of hyperspectral images," Proc. IEEE, vol. 101, no. 3, pp. 652-675, Mar. 2013.

[7] R. L. Kettig and D. A. Landgrebe, "Classification of multispectral image data by extraction and classification of homogeneous objects," IEEE Trans. Geosci. Electron., vol. 14, no. 1, pp. 19-26, Jan. 1976.

[8] Y. Wang, K. Loe, T. Tan, and J. Wu, "A dynamic hidden markov random field model for foreground and shadow segmentation," in Proc. WACV, vol. 1, Jan. 2005, pp. 474-480.

[9] X. Cao, F. Zhou, L. Xu, D. Meng, Z. Xu, and J. Paisley, "Hyperspectral image classification with markov random fields and a convolutional neural network," IEEE Trans. Image Process., vol. 27, no. 5, pp. 2354 2367, May. 2018.

[10] B. Zhang, S. Li, X. Jia, L. Gao, and M. Peng, "Adaptive markov random field approach for classification of hyperspectral imagery," IEEE Geosci. Remote Sens. Lett., vol. 8, no. 5, pp. 973-977, Sep. 2011.

[11] G. Moser and S. B. Serpico, "Combining support vector machines and markov random fields in an integrated framework for contextual image classification," IEEE Trans. Geosci. Remote Sens., vol. 51, no. 5, pp. 2734-2752, May. 2013.

[12] J. A. Benediktsson, J. A. Palmason, and J. R. Sveinsson, "Classification of hyperspectral data from urban areas based on extended morphological profiles," IEEE Trans. Geosci. Remote Sens., vol. 43, no. 3, pp. 480-491, Mar. 2005.

[13] L. Shen and S. Jia, "Three-dimensional gabor wavelets for pixel-based hyperspectral imagery classification," IEEE Trans. Geosci. Remote Sens., vol. 49 , no. 12 , pp. 5039-5046, Dec. 2011.

[14] G. Camps-Valls, L. Gomez-Chova, J. Munoz-Mari, J. Vila-Frances, and J. Calpe-Maravilla, "Composite kernels for hyperspectral image classification," IEEE Geosci. Remote Sens. Lett., vol. 3, no. 1, pp. 93-97, Jan. 2006.

[15] L. Fang, S. Li, W. Duan, J. Ren, and J. A. Benediktsson, "Classification of hyperspectral images by exploiting spectral-spatial information of superpixel via multiple kernels," IEEE Trans. Geosci. Remote Sens., vol. 53, no. 12, pp. 6663-6674, Dec. 2015.

[16] Y. Xu, Z. Wu, and Z. Wei, "Spectral-spatial classification of hyperspectral image based on low-rank decomposition," IEEE J. Sel. Topics Appl. Earth Observ. Remote Sens., vol. 8, no. 6, pp. 2370-2380, Jun. 2015.

[17] T. N. Kipf and M. Welling, "Semi-supervised classification with graph convolutional networks," in Proc. ICLR, 2017.

[18] X. Wang, R. Girshick, A. Gupta, and K. He, "Non-local neural networks," pp. 7794-7803, Jun. 2017.

[19] Y. Li and A. Gupta, "Beyond grids: Learning graph representations for visual recognition," in Proc. Adv. Neural Inf. Process. Syst., 2018, pp. 9225-9235.

[20] W. Luo, Y. Li, R. Urtasun, and R. Zemel, "Understanding the effective receptive field in deep convolutional neural networks," pp. 4898-4906, 2016.

[21] R. Li, S. Wang, F. Zhu, and J. Huang, "Adaptive graph convolutional neural networks," in Proc. AAAI, 2018.

[22] A. Villa, J. A. Benediktsson, J. Chanussot, and C. Jutten, "Hyperspectral image classification with independent component discriminant analysis," IEEE Trans. Geosci. Remote Sens., vol. 49, no. 12, pp. 4865-4876, Dec. 2011.

[23] L. Zhang, Q. Zhang, B. Du, X. Huang, Y. Y. Tang, and D. Tao, "Simultaneous spectral-spatial feature selection and extraction for hyperspectral images," IEEE Trans. Cybern., vol. 48, no. 1, pp. 16-28, Jan. 2018.

[24] K. Liu, Y. Lin, and C. Chen, "Linear spectral mixture analysis via multiple-kernel learning for hyperspectral image classification," IEEE Trans. Geosci. Remote Sens., vol. 53, no. 4, pp. 2254-2269, Apr. 2015.

[25] Y. Zhou, J. Peng, and C. L. P. Chen, "Extreme learning machine with composite kernels for hyperspectral image classification," IEEE J. Sel. Topics Appl. Earth Observ. Remote Sens., vol. 8, no. 6, pp. 2351-2360, Jun. 2015.

[26] X. Huang and L. Zhang, "An adaptive mean-shift analysis approach for object extraction and classification from urban hyperspectral imagery," IEEE Trans. Geosci. Remote Sens., vol. 46, no. 12, pp. 4173-4185, Dec. 2008.

[27] Y. Y. Tang, Y. Lu, and H. Yuan, "Hyperspectral image classification based on three-dimensional scattering wavelet transform," IEEE Trans. Geosci. Remote Sens., vol. 53, no. 5, pp. 2467-2480, May. 2015.

[28] J. Zabalza, J. Ren, J. Zheng, J. Han, H. Zhao, S. Li, and S. Marshall, "Novel two-dimensional singular spectrum analysis for effective feature extraction and data classification in hyperspectral imaging," IEEE Trans. Geosci. Remote Sens., vol. 53, no. 8, pp. 4418-4433, Aug. 2015.
[29] J. Peng, Y. Zhou, and C. L. P. Chen, "Region-kernel-based support vector machines for hyperspectral image classification," IEEE Trans. Geosci. Remote Sens., vol. 53, no. 9, pp. 4810-4824, Sep. 2015.

[30] J. Xia, M. Dalla Mura, J. Chanussot, P. Du, and X. He, "Random subspace ensembles for hyperspectral image classification with extended morphological attribute profiles," IEEE Trans. Geosci. Remote Sens. vol. 53, no. 9, pp. 4768-4786, Sep. 2015.

[31] M. Pesaresi and J. A. Benediktsson, "A new approach for the morphological segmentation of high-resolution satellite imagery," IEEE Trans. Geosci. Remote Sens., vol. 39, no. 2, pp. 309-320, Feb. 2001.

[32] M. Fauvel, J. A. Benediktsson, J. Chanussot, and J. R. Sveinsson, "Spectral and spatial classification of hyperspectral data using SVMs and morphological profiles," IEEE Trans. Geosci. Remote Sens., vol. 46, no. 11, pp. 3804-3814, Nov. 2008.

[33] M. Dalla Mura, J. A. Benediktsson, B. Waske, and L. Bruzzone, "Morphological attribute profiles for the analysis of very high resolution images," IEEE Trans. Geosci. Remote Sens., vol. 48, no. 10, pp. 37473762, Oct. 2010.

[34] M. Dalla Mura, A. Villa, J. A. Benediktsson, J. Chanussot, and L. Bruzzone, "Classification of hyperspectral images by using extended morphological attribute profiles and independent component analysis," IEEE Trans. Geosci. Remote Sens., vol. 8, no. 3, pp. 542-546, May. 2011.

[35] M. Pedergnana, P. R. Marpu, M. D. Mura, J. A. Benediktsson, and L. Bruzzone, "A novel technique for optimal feature selection in attribute profiles based on genetic algorithms," IEEE Trans. Geosci. Remote Sens., vol. 51, no. 6, pp. 3514-3528, Jun. 2013.

[36] J. Li, H. Zhang, and L. Zhang, "Efficient superpixel-level multitask joint sparse representation for hyperspectral image classification," IEEE Trans. Geosci. Remote Sens., vol. 53, no. 10, pp. 5338-5351, Oct. 2015.

[37] G. Zhang, X. Jia, and J. Hu, "Superpixel-based graphical model for remote sensing image mapping," IEEE Trans. Geosci. Remote Sens., vol. 53, no. 11, pp. 5861-5871, Nov. 2015.

[38] B. Cui, X. Xie, X. Ma, G. Ren, and Y. Ma, "Superpixel-based extended random walker for hyperspectral image classification," IEEE Trans. Geosci. Remote Sens., vol. 56, no. 6, pp. 3233-3243, Jun. 2018.

[39] W. Hamilton, Z. Ying, and J. Leskovec, "Inductive representation learning on large graphs," in Proc. Adv. Neural Inf. Process. Syst., 2017, pp. 1024-1034.

[40] P. Veličković, G. Cucurull, A. Casanova, A. Romero, P. Lio, and Y. Bengio, "Graph attention networks," arXiv preprint arXiv:1710.10903, 2017.

[41] J. Bruna, W. Zaremba, A. Szlam, and Y. LeCun, "Spectral networks and locally connected networks on graphs," arXiv preprint arXiv:1312.6203, 2013.

[42] M. Defferrard, X. Bresson, and P. Vandergheynst, "Convolutional neural networks on graphs with fast localized spectral filtering," in Proc. Adv. Neural Inf. Process. Syst., 2016, pp. 3844-3852.

[43] T. Lei and H. Liu, "Relational learning via latent social dimensions," in Proc. ACM SIGKDD. ACM, 2009, pp. 817-826.

[44] K. C. P. E. W. L. H. R. Ying, R. He and J. Leskovec, "Graph convolutional neural networks for web-scale recommender systems," in Proc. ACM SIGKDD. ACM, 2018, pp. 974-983.

[45] H. Zhuang, C. Wang, C. Li, Q. Wang, and X. Zhou, "Natural language processing service based on stroke-level convolutional networks for chinese text classification," in Proc. IEEE ICWS, Jun. 2017, pp. 404411.

[46] Y. Zhang, S. Song, E. Yumer, M. Savva, J. Lee, H. Jin, and T. Funkhouser, "Physically-based rendering for indoor scene understanding using convolutional neural networks," in Proc. IEEE CVPR, Jul. 2017, pp. 5057-5065.

[47] A. Qin, Z. Shang, J. Tian, Y. Wang, T. Zhang, and Y. Y. Tang, "Spectralspatial graph convolutional networks for semisupervised hyperspectral image classification," IEEE Geosci. Remote Sens. Lett., vol. 16, no. 2, pp. 241-245, Feb. 2019.

[48] Z. Hao, Z. Yang, W. Liu, J. Liang, and Y. Li, "Improving deep neural networks using softplus units," in Proc. IJCNN, Jul. 2015, pp. 1-4.

[49] D. K. Hammond, P. Vandergheynst, and R. Gribonval, "Wavelets on graphs via spectral graph theory," Appl. Comput. Harmon. Anal., vol. 30, no. 2, pp. 129-150, Dec. 2011.

[50] R. Achanta, A. Shaji, K. Smith, A. Lucchi, P. Fua, and S. Ssstrunk, "Slic superpixels compared to state-of-the-art superpixel methods," IEEE Trans. Pattern Anal. Mach. Intell., vol. 34, no. 11, pp. 2274-2282, Nov. 2012.

[51] X. Yang, Y. Ye, X. Li, R. Y. K. Lau, X. Zhang, and X. Huang, "Hyperspectral image classification with deep learning models," IEEE Trans. Geosci. Remote Sens., vol. 56, no. 9, pp. 5408-5423, Sep. 2018. 
[52] W. Li, G. Wu, F. Zhang, and Q. Du, "Hyperspectral image classification using deep pixel-pair features," IEEE Trans. Geosci. Remote Sens., vol. 55, no. 2, pp. 844-853, Feb. 2017.

[53] J. Li, X. Huang, P. Gamba, J. M. Bioucas-Dias, L. Zhang, J. A Benediktsson, and A. Plaza, "Multiple feature learning for hyperspectral image classification," IEEE Trans. Geosci. Remote Sens., vol. 53, no. 3, pp. 1592-1606, Mar. 2015.

[54] C. Bo, H. Lu, and D. Wang, "Hyperspectral image classification via jcr and svm models with decision fusion," IEEE Geosci. Remote Sens. Lett., vol. 13, no. 2, pp. 177-181, Feb. 2016.

[55] H. Gao, Z. Wang, and S. Ji, "Large-scale learnable graph convolutional networks," in Proc. SIGKDD. ACM, 2018, pp. 1416-1424.

[56] C. Gong, T. Liu, D. Tao, K. Fu, E. Tu, and J. Yang, "Deformed graph laplacian for semisupervised learning," IEEE Trans. Neural Netw. Learn. Syst., vol. 26, no. 10, pp. 2261-2274, Oct. 2015. 